\title{
Correlation of spin and velocity in the homogeneous cooling state of a granular gas of rough particles
}

\author{
W.T. Kranz ${ }^{1,2}$, N.V. Brilliantov ${ }^{3}$, T. Pöschel ${ }^{4,5}$, and A. Zippelius ${ }^{1,2}$ \\ 1 Max-Planck-Institute for Dynamics and Self Organization, Bunsenstr. 10, 37073 Göttingen, Germany \\ 2 Institute of Theoretical Physics, University of Göttingen, Friedrich-Hund-Platz 1, \\ 37077 Göttingen, Germany \\ 3 Department of Mathematics, University of Leicester, University Road, Leicester LE1 7RH, UK \\ 4 Cluster of Excellence "Engineering of Advanced Materials", Friedrich-Alexander-University, \\ Erlangen, Germany \\ 5 Universität Erlangen-Nürnberg, Institute for Multiscale Simulation, Nägelsbachstrasse 49b, \\ Erlangen, Germany
}

\begin{abstract}
In a granular gas of rough particles the spin of a grain is correlated with its linear velocity. We develop an analytical theory to account for these correlations and compare its predictions to numerical simulations, using Direct Simulation Monte Carlo as well as Molecular Dynamics. The system is shown to relax from an arbitrary initial state to a steady-state, which is characterized by time-independent, finite correlations of spin and linear velocity. The latter are analyzed systematically for a wide range of system parameters, including the coefficients of tangential and normal restitution as well as the moment of inertia of the particles. For most parameter values the axis of rotation and the direction of linear momentum are perpendicular like in a sliced tennis ball, while parallel orientation, like in a rifled bullet, occurs only for a small range of parameters. The limit of smooth spheres is singular: any arbitrarily small roughness unavoidably causes significant translation-rotation correlations, whereas for perfectly smooth spheres the rotational degrees of freedom are completely decoupled from the dynamic evolution of the gas.
\end{abstract}

\section{Introduction}

Materials which are composed of macroscopic objects, i.e. granular media, attract increasing scientific interest due to their importance in nature and technology, e.g. [1,2]. The latter may be exemplified by transport and storage of sand, cereals, granular chemicals, etc. the formerby avalanches, land slides, dust devils, etc. Spectacular celestial objects, like planetary rings or interstellar dust clouds, can serve as another example of natural granular systems [3]. The granular matter exists there in a gaseous state and exhibits many properties of a common molecular gas, e.g. $[1,4-6]$. The main (and very important) difference between a granular gas and a molecular gas is the dissipative nature of particle interactions, which are described by the macroscopic mechanics of solids rather than by a microscopic interaction potential. The consequences of the dissipative interactions are quite substantial: A spatially homogeneous state is unstable [7-9], velocities are not distributed according to a Maxwell-Boltzmann distribution [10-18] and the diffusion or self-diffusion is anomalous [19-23]. These properties of a granular gas have been observed for the case of smooth particles, when grain collisions do not affect their rotational motion. This is, certainly, an oversimplified model, since real grains have a rough surface and exchange rotational and translational energy in collisions. 
Real granular particles experience frictional forces when colliding. Hence, a more adequate model takes into account the rotational motion of particles and the exchange of rotational and translational energy in collisions $[1,6,10,24-35]$. Dissipative frictional gases exhibit additional unusual features which are not present in molecular gases. For instance, equipartition between rotational and translational motion does not hold [24] and the hydrodynamic description requires an additional field and a dynamic equation to account for its evolution $[26,36]$. Moreover, the rotational and translational motion of particles are correlated as mentioned in a very implicit way in Appendix E of [26] and worked out in [37]. In the present study we analyze the latter effect in detail.

In Section 2 we introduce a model of frictional particles and the dynamical variables of interest. Subsequently in Section 3 an approximate theory is developed and in Section 4 we briefly explain the simulation techniques. The main results are presented in Section 5, where we compare predictions of the analytical theory with data from simulations. The emphasis lies on the correlations in the steady state, but we also briefly discuss the relaxation to the steady state. The technical details of the calculations are given in the Appendix.

\section{Model and observables}

We consider a granular gas consisting of $N$ inelastic hard spheres of radius $a$, mass $m$, and moment of inertia $I=q m a^{2}$. Here the dimensionless variable $\mathrm{q}$ is determined by the mass distribution within the particle. The state of the system is fully described by the particles' positions $\left\{\mathbf{r}_{i}\right\}$, velocities $\left\{\mathbf{v}_{i}\right\}$, and angular velocities $\left\{\boldsymbol{\omega}_{i}\right\}$ for $i=1, \ldots, N$. The particles move freely in between instantaneous collisions, whereupon their linear and angular velocities change according to the collision rule. The relative velocity at the point of contact of colliding particles is

$$
\mathbf{g} \equiv \mathbf{v}_{1}-\mathbf{v}_{2}+a \hat{\mathbf{n}} \times\left(\boldsymbol{\omega}_{1}+\boldsymbol{\omega}_{2}\right)
$$

with $\hat{\mathbf{n}} \equiv \hat{\mathbf{n}}_{12} \equiv\left(\mathbf{r}_{1}-\mathbf{r}_{2}\right) /\left|\mathbf{r}_{1}-\mathbf{r}_{2}\right|$. The post-collisional (primed) velocity is related to the pre-collisional one by

$$
\mathbf{g}^{\prime} \cdot \hat{\mathbf{n}}=-\varepsilon_{n} \mathbf{g} \cdot \hat{\mathbf{n}} \quad \text { and } \quad \mathbf{g}^{\prime} \times \hat{\mathbf{n}}=\varepsilon_{t} \mathbf{g} \times \hat{\mathbf{n}} .
$$

The coefficient of normal restitution is denoted by $\varepsilon_{n}$ with $0 \leq \varepsilon_{n} \leq 1$. The value $\varepsilon_{n}=0$ implies no relative motion in the normal direction after the collision, whereas for $\varepsilon_{n}=1$ no dissipation of the normal component of the relative motion occurs. The coefficient of tangential restitution has two elastic limits, namely $\varepsilon_{t}=1$ corresponding to smooth spheres and $\varepsilon_{t}=-1$ corresponding to perfectly rough (reflecting) collisions without loss of energy for the tangential motion. For all other values energy is lost in the tangential component. In general, both coefficients of restitution, $\varepsilon_{n}$ and $\varepsilon_{t}$, depend on the impact velocity [38-41].

Together with the conservation of linear and angular momentum the collision rule, Eq. (2), determines the post-collisional velocities in terms of the pre-collisional ones:

$$
\mathbf{v}_{1}^{\prime}=\mathbf{v}_{1}-\boldsymbol{\delta}, \quad \boldsymbol{\omega}_{1}^{\prime}=\boldsymbol{\omega}_{1}+\frac{1}{q a}(\hat{\mathbf{n}} \times \boldsymbol{\delta}), \quad \mathbf{v}_{2}^{\prime}=\mathbf{v}_{2}+\boldsymbol{\delta}, \quad \boldsymbol{\omega}_{2}^{\prime}=\boldsymbol{\omega}_{2}+\frac{1}{q a}(\hat{\mathbf{n}} \times \boldsymbol{\delta})
$$

where $m \boldsymbol{\delta}$ denotes the exchange of linear momentum with

$$
\begin{aligned}
\boldsymbol{\delta} & \equiv \eta_{t} \mathbf{g}+\left(\eta_{n}-\eta_{t}\right)(\hat{\mathbf{n}} \cdot \mathbf{g}) \hat{\mathbf{n}} \\
\eta_{n} & \equiv \frac{1+\varepsilon_{n}}{2}, \quad \eta_{t} \equiv \frac{q}{2} \frac{1-\varepsilon_{t}}{1+q} .
\end{aligned}
$$

In the present study we address only non-driven systems. Moreover, we focus on the homogeneous cooling state (HCS) of a gas, which is characterized by two time-dependent granular 
temperatures, one for the translational and one for the rotational motion,

$$
T=\frac{m}{3 N} \sum_{i=1}^{N} \mathbf{v}_{i}^{2} \quad \text { and } \quad R=\frac{I}{3 N} \sum_{i=1}^{N} \boldsymbol{\omega}_{i}^{2} .
$$

One generally observes that after a transient period the system reaches a steady-state where $r \equiv R(t) / T(t)=$ const., that is, both temperatures decay with the same rate. In general, $r \neq 1$ so that equipartition is violated. The value of $r$ depends on the collision parameters as well as on the moment of inertia $[24,42]$.

In this paper we focus on the correlation between the axis of rotation of a granular particle and the direction of its linear velocity, which may be quantified by the angle $\theta_{i}$ between the linear and rotational velocity,

$$
\cos \theta_{i}=\frac{\mathbf{v}_{i} \cdot \boldsymbol{\omega}_{i}}{\left|\mathbf{v}_{i}\right|\left|\boldsymbol{\omega}_{i}\right|}
$$

All information on the angle is contained in the distribution

$$
f(\cos \theta)=\frac{1}{N} \sum_{i=1}^{N} \delta\left(\cos \theta-\cos \theta_{i}\right) .
$$

In a molecular gas all values of $\cos \theta$ occur with equal probability due to equipartition. In contrast for a granular gas we know that equipartition is violated and we expect to observe deviations from the equi-distribution.

Because of symmetry, the average of $\cos \theta_{i}$ over all particles vanishes. Thus, a measure of correlations is the second moment,

$$
\left\langle\cos ^{2} \theta\right\rangle=\frac{1}{N} \sum_{i} \frac{\left(\mathbf{v}_{i} \cdot \boldsymbol{\omega}_{i}\right)^{2}}{\mathbf{v}_{i}^{2} \boldsymbol{\omega}_{i}^{2}} .
$$

If the angular and linear velocities are not correlated in their direction, $\left\langle\cos ^{2} \theta\right\rangle=1 / 3$. Hence, any deviation of $\left\langle\cos ^{2} \theta\right\rangle$ from $1 / 3$ indicates correlations. Moreover, if $\left\langle\cos ^{2} \theta\right\rangle<1 / 3$ the angular and linear velocities are preferably perpendicular, like in a sliced tennis ball, while for $\left.\left\langle\cos ^{2} \theta\right\rangle\right\rangle$ $1 / 3$ they are preferably aligned like in a rifled bullet.

\section{Approximate theory: Low order moments}

Evolution of a granular gas is traditionally described by the Boltzmann equation for the one-particle distribution function $f(\mathbf{v}, \boldsymbol{\omega}, t)$ which reads for homogeneous systems addressed here,

$$
\frac{\partial}{\partial t} f(\mathbf{v}, \boldsymbol{\omega}, t)=I(f, f)
$$

The collision integral $I(f, f)$ accounts for the alteration of the distribution function $f(\mathbf{v}, \boldsymbol{\omega}, t)$ in the pair-wise collisions and is completely determined by the collision rule (3). The above kinetic equation is very complicated and can hardly be solved in general. The standard approach, used in kinetic theories (the "Grad-like" procedure) is the following: (i) assume a form of the distribution function, which depends on a limited number of moments, e.g. $\left\langle m v^{2}\right\rangle,\left\langle I \omega^{2}\right\rangle$, $\left\langle(\mathbf{v} \cdot \boldsymbol{\omega})^{2}\right\rangle$, etc., (ii) substitute this form into the kinetic equation (10) and (iii) get a closed set of equations for these moments ${ }^{1}$. Computations become more straightforward and notations more compact if the formalism of the pseudo-Liouville operator is used; we briefly outline it below.

\footnotetext{
${ }^{1}$ Generally, one can assume the dependence of $f(\mathbf{v}, \boldsymbol{\omega}, t)$ on some free parameters (not necessarily moments) and derive the set of equations for these parameters, projecting the Boltzmann equation onto the low order moments.
} 
The evolution of any dynamical variable

$$
F(t)=F\left(\left\{\mathbf{r}_{i}(t), \mathbf{v}_{i}(t), \boldsymbol{\omega}_{i}(t)\right\}\right)
$$

may be obtained by means of the pseudo-Liouville operator $\mathcal{L}_{+}$via

$$
\partial_{t} F(t)=i \mathcal{L}_{+} F(t) \text { for } t>0 .
$$

For hard spheres the pseudo-Liouville operator decomposes into two parts, $\mathcal{L}_{+}=\mathcal{L}_{0}+\mathcal{L}_{+}^{\prime}$, where $\mathcal{L}_{0}=\mathcal{L}_{0}^{\text {tr }}+\mathcal{L}_{0}^{\text {rot }}$ describes the free streaming of translational and rotational motion of particles. Here $\mathcal{L}_{0}^{\text {tr }}=\sum_{i} \mathbf{v}_{i} \cdot \nabla_{i}$ and a similar expression for $\mathcal{L}_{0}^{\text {rot }}$. The latter is not needed here, because we never specify the orientation of our particles, which are perfect spheres. The interaction part of the pseudo-Liouville operator reads, $\mathcal{L}_{+}^{\prime}=\sum_{i<j} \mathcal{T}_{i j}$, where the binary collision operator $\mathcal{T}_{i j}$ reads $[11,24]$

$$
i \mathcal{T}_{i j}=-\hat{\mathbf{n}}_{i j} \cdot \mathbf{v}_{i j} \Theta\left(-\hat{\mathbf{n}}_{i j} \cdot \mathbf{v}_{i j}\right) \delta\left(r_{i j}-2 a\right)\left(\hat{b}_{i j}-1\right)
$$

The operator $\hat{b}_{i j}$ replaces unprimed by primed values according to the collision rule, Eq. (3). For example,

$$
\hat{b}_{12} \mathbf{v}_{1}=\mathbf{v}_{1}^{\prime}, \quad \hat{b}_{12} \mathbf{v}_{2}=\mathbf{v}_{2}^{\prime}, \quad \hat{b}_{12} \mathbf{v}_{k}=\mathbf{v}_{k}, \quad k \neq 1,2
$$

with $\mathbf{v}_{1}^{\prime}$ and $\mathbf{v}_{2}^{\prime}$ given by Eq. (3) and with similar relations for the rotational velocities.

The ensemble average of a dynamic variable is defined by

$$
\langle F\rangle_{t}=\int \mathrm{d} \Gamma \rho(0) F(t)=\int \mathrm{d} \Gamma \rho(t) F(0)
$$

with $\mathrm{d} \Gamma=\prod_{i}\left(d^{3} r_{i} d^{3} v_{i} d^{3} \omega_{i}\right)$. Here $F(t)=\exp \left(-i \mathcal{L}_{+} t\right) F(0)$ and $\rho(t)=\exp \left(-i \mathcal{L}_{+}^{\dagger} t\right) \rho(0)$ denotes the $N$-particle distribution, whose evolution is governed by the adjoint $\mathcal{L}_{+}^{\dagger}$ of the evolution operator $\mathcal{L}_{+}$. Differentiating Eq. (15) one obtains

$$
\begin{aligned}
\frac{\mathrm{d}}{\mathrm{d} t}\langle F\rangle_{t} & =\int \mathrm{d} \Gamma \rho(0) \frac{\mathrm{d}}{\mathrm{d} t} F(t)=\int \mathrm{d} \Gamma \rho(0) i \mathcal{L}_{+} F(t)=\int \mathrm{d} \Gamma \rho(0) \exp \left(i \mathcal{L}_{+} t\right) i \mathcal{L}_{+} F(0) \\
& =\int \mathrm{d} \Gamma \rho(t) i \mathcal{L}_{+} F(0)=\left\langle i \mathcal{L}_{+} F\right\rangle_{t}
\end{aligned}
$$

It is impossible to compute the time-dependent $N$-particle distribution exactly, so that we have to resort to approximations. A standard procedure in the analytical treatment of granular gases is to assume homogeneity and molecular chaos, e.g. [6] (see also [43]). Under these assumptions the $N$-particle velocity distribution function takes the form

$$
\rho(t)=g_{N}\left(\mathbf{r}_{1}, \ldots, \mathbf{r}_{N}\right) \prod_{i} f\left(\mathbf{v}_{i}, \boldsymbol{\omega}_{i}, t\right),
$$

where the $N$-particle correlation function of a hard sphere system, $g_{N}\left(\mathbf{r}_{1}, \ldots, \mathbf{r}_{N}\right)$, is not affected by the particle roughness. For the HCS it may be approximated by the corresponding function of an equilibrium hard-sphere system (e.g. [6]). For an isotropic system $f(\mathbf{v}, \boldsymbol{\omega})$ depends in general on $v=|\mathbf{v}|, \boldsymbol{\omega}=|\boldsymbol{\omega}|$ and the angle $\theta(\cos \theta=\mathbf{v} \cdot \boldsymbol{\omega} /(|\mathbf{v}||\boldsymbol{\omega}|)$. Here we are particularly interested in the dependence on $\cos \theta$ and expand $f$ around a product of Maxwellians in Legendre polynomials $P_{q}(\cos \theta)$

$$
f(\mathbf{v}, \boldsymbol{\omega}, t) \propto \exp \left(-\frac{m \mathbf{v}^{2}}{2 T(t)}\right) \exp \left(-\frac{I \boldsymbol{\omega}^{2}}{2 R(t)}\right) \sum_{n=0}^{\infty} \sum_{p=0}^{\infty} \sum_{q=0}^{\infty} b_{n p q}(t) \mathbf{v}^{2 n} \boldsymbol{\omega}^{2 p} P_{q}(\cos \theta)
$$

where $b_{n p q}(t)$ are time dependent expansion coefficients and the distribution function has to be normalized according to $\int d \mathbf{v} d \boldsymbol{\omega} f=1$. In the following we use a simple Gaussian even though the distributions are non-Gaussian for strong dissipation and high densities. Deviations 
have been handled by an expansion in Sonine polynomials [44]. Here we concentrate on the dependence on $\cos \theta$ and leave a more general Ansatz with both, angular correlations and non-Gaussian distributions, to future work. To keep the calculations tractable, we limit the calculation to the lowest non-trivial order

$$
f(\mathbf{v}, \boldsymbol{\omega}, t) \propto \exp \left(-\frac{m \mathbf{v}^{2}}{2 T(t)}\right) \exp \left(-\frac{I \boldsymbol{\omega}^{2}}{2 R(t)}\right)\left[1+b(t) \mathbf{v}^{2} \boldsymbol{\omega}^{2} P_{2}(\cos \theta)\right]
$$

where $b(t) \equiv b_{112}(t)$ and $P_{2}(\cos \theta)=3 / 2\left(\cos ^{2} \theta-1 / 3\right)$. The terms for odd $q$ vanish by symmetry.

The lowest order coefficient $b(t)$ is simply related to the quantity of interest $\left\langle\cos ^{2} \theta\right\rangle_{t}$. Using $P_{0}(\cos \theta)=1$ and expressing $\left\langle\cos ^{2} \theta\right\rangle_{t}$ in terms of Legendre polynomials we can write

$$
\left\langle\cos ^{2} \theta\right\rangle_{t}=\frac{1}{3} \int_{v} \int_{\omega}\left[P_{0}(\cos \theta)+2 P_{2}(\cos \theta)\right]\left[P_{0}(\cos \theta)+b(t) v^{2} \omega^{2} P_{2}(\cos \theta)\right]
$$

where for brevity we introduce the shorthand notation

$$
\int_{v}=\left(\frac{m}{2 \pi T}\right)^{3 / 2} \int \mathrm{d}^{3} v \exp \left(-\frac{m v^{2}}{2 T}\right)
$$

and similarly for $\int_{\omega}$. The angular integration in the Eq. (20) may be performed using the orthogonality relation for Legendre polynomials, yielding

$$
\left\langle\cos ^{2} \theta\right\rangle_{t}=\frac{1}{3}+b(t) \frac{6 T(t) R(t)}{5 q m^{2} a^{2}} .
$$

Hence, the correlations of interest manifest themselves through the coefficient $b(t)$ - the larger the coefficient, the more pronounced are deviations from the value $\left\langle\cos ^{2} \theta\right\rangle=1 / 3$ of the uncorrelated case.

To summarize our analytical approach so far: The time dependent $N$-particle distribution has been parametrised by three time-dependent functions $T(t), R(t)$ and $b(t)$, which have to be calculated self-consistently. This is achieved by applying the general equation (16) for the evolution of a dynamical variable to $T(t), R(t)$ and $b(t)$ and using our Ansatz for $\rho(t)$, see Eqs. (17), (19). Due to the specific functional form of our ansatz - a product of polynomials and gaussians in the velocities - the set of self-consistent equations can be determined analytically. The detailed derivation of these equations are given in the Appendix.

The result of these cumbersome (although straightforward) calculations are three first order differential equations for $T(t), R(t)$ and $b(t)$. These simplify, if we measure times in units of the Enskog collision frequency $\omega_{E}=16(\pi T / m)^{1 / 2} n a^{2} g_{2}(2 a)$. In other words we rescale time according to $d \tau=\omega_{E} d t$ and obtain:

$$
\begin{aligned}
& \frac{\mathrm{d} T}{\mathrm{~d} \tau}=-A T(\tau)+B\left[1-\frac{b(\tau)}{2} \frac{T(\tau) R(\tau)}{q m^{2} a^{2}}\right] R(\tau) \\
& \frac{\mathrm{d} R}{\mathrm{~d} \tau}=B T(\tau)-C\left[1-\frac{b(\tau)}{2} \frac{T(\tau) R(\tau)}{q m^{2} a^{2}}\right] R(\tau)
\end{aligned}
$$

where

$$
A \equiv \eta_{n}\left(1-\eta_{n}\right)+\eta_{t}\left(1-\eta_{t}\right), \quad B \equiv \frac{\eta_{t}^{2}}{q}, \quad C \equiv \frac{\eta_{t}}{q}\left(1-\frac{\eta_{t}}{q}\right)
$$

and

$$
\begin{aligned}
20 \frac{\mathrm{d} b}{\mathrm{~d} \tau}= & -b(\tau)\left[A^{(1)}+B^{(1)} \frac{R(\tau)}{T(\tau)}+\frac{40}{T(\tau)} \frac{\mathrm{d} T}{\mathrm{~d} \tau}(\tau)+\frac{40}{R(\tau)} \frac{\mathrm{d} R}{\mathrm{~d} \tau}(\tau)\right] \\
& -\frac{q m^{2} a^{2}}{T(\tau) R(\tau)}\left[A^{(0)}+B^{(0)} \frac{R(\tau)}{T(\tau)}+C^{(0)} \frac{T(\tau)}{R(\tau)}\right] .
\end{aligned}
$$


The constants are given by:

$$
\begin{gathered}
A^{(0)} \equiv \frac{16}{3} \frac{\eta_{t}^{3}}{q}\left(\frac{2 \eta_{t}}{q}-1\right)-\frac{2}{3} \frac{\eta_{t}^{2}}{q}\left(\frac{8 \eta_{t}}{q}-3\right)+\frac{1}{3} \frac{\eta_{t}}{q}\left(\frac{\eta_{t}}{q}-1\right)+\frac{8}{3} \frac{\eta_{t}}{q}\left(\frac{\eta_{t}}{q}-1\right) \eta_{n}\left(\eta_{n}-1\right) \\
B^{(0)} \equiv \frac{1}{3} \frac{\eta_{t}^{2}}{q}\left[\frac{16 \eta_{t}}{q}\left(\frac{\eta_{t}}{q}-1\right)+5\right] \\
C^{(0)} \equiv \frac{2}{3} \frac{\eta_{t}^{2}}{q}\left[8 \eta_{t}\left(\eta_{t}-1\right)+4 \eta_{n}\left(\eta_{n}-1\right)+3\right] \\
A^{(1)} \equiv-\frac{8 \eta_{t}^{3}}{q}\left(\frac{2 \eta_{t}}{q}-1\right)+\frac{1}{3} \frac{\eta_{t}^{2}}{q}\left(\frac{24 \eta_{t}}{q}-37\right)-\frac{5}{6} \frac{\eta_{t}}{q}\left(\frac{9 \eta_{t}}{q}-29\right)-\frac{4 \eta_{t} \eta_{n}^{2}}{q}\left(\frac{\eta_{t}}{q}-1\right) \\
+\frac{4}{3} \frac{\eta_{t} \eta_{n}}{q}\left(\frac{3 \eta_{t}}{q}-14\right)-12 \eta_{t} \eta_{n}+22\left(\eta_{t}+\eta_{n}\right)-6\left(\eta_{t}^{2}+\eta_{n}^{2}\right) \\
B^{(1)} \equiv-\frac{2}{3} \frac{\eta_{t}^{2}}{q}\left[\frac{8 \eta_{t}}{q}\left(\frac{\eta_{t}}{q}-1\right)+1\right]
\end{gathered}
$$

Equations (23) and (25) constitute a set of self-consistent equations for the observables $T(t), R(t)$, and $b(t)$. Note that applying the technique of the pseudo-Liouville operator we essentially use the Grad-like procedure, that is, we assume the form of the distribution function (19) with three parameters, related to the moments of the distribution function and find the equations for these parameters.

\section{Simulations}

We performed both Direct Simulation Monte Carlo (DSMC) [45] and event-driven Molecular Dynamics (MD) [46] calculations to check the predictions of the analytical theory. DSMC determines the stationary distribution of the scaled velocities by numerically solving the kinetic Boltzmann equation which is based on the assumption of molecular chaos. Consequently, for its application it is assumed that the gas is uniform, thus, spatial correlations of the particles are neglected. If this precondition is given, that is, if the spatial correlations are negligible, DSMC yields very precise statistical results because of the large number of particles which can be simulated (here we use $N=2 \times 10^{7}$ particles $^{2}$ ).

Molecular Dynamics calculates the trajectories of the particles using the collision rule, Eq. (2), therefore, MD allows to trace the evolution of the correlation. On the other hand, MD is restricted to much smaller systems as compared to DSMC. Although MD is free from the mentioned assumptions, DSMC is significantly more efficient for a homogeneous granular gas. Moreover, in the limit of low density both methods provide, in principle, identical results for the steady-state [46]. In practice, we use MD for $N=8000$ particles to study the transient process of the system's relaxation to its steady-state and up to $N=10^{5}$ for steady state correlations. The volume fraction was $\phi_{0}=\frac{N}{V} \frac{4 \pi a^{3}}{3}=0.0146$ or even smaller, such that the size of the system $L=(4 \pi N / 3)^{1 / 3} a$ was small enough to remain always in the HCS.

2 To be precise, although the mathematical operations in DSMC look like a particle simulation, the particles in the simulation do not correspond to real particles. They are better considered as quanta of probability [47]. 


\section{Results}

Starting from a random distribution of velocities and angular velocities with mean $\langle\mathbf{v}\rangle=\langle\boldsymbol{\omega}\rangle=$ 0 , after some transient period the system relaxes to a steady state where the correlation of the spin and the translational velocity as well as the ratio of translational and rotational temperatures reach stationary values. We quantify these correlations by means of the second moment $\left\langle\cos ^{2} \theta\right\rangle$, see Eq. (9) and analyze this quantity as a function of three parameters, $\varepsilon_{n}, \varepsilon_{t}$, and $q$ in Section 5.1. The relaxation to the steady state is discussed in Section 5.2 and in Section 5.3 we consider correlations beyond the second moment and investigate the distribution of $\cos \theta$.

\subsection{Steady-state correlations}

To study the steady-state properties it is convenient to introduce an auxiliary variable

$$
x(\tau) \equiv b(\tau) \frac{T(\tau) R(\tau)}{q m^{2} a^{2}}=\frac{5}{6}\left(\left\langle\cos ^{2} \theta\right\rangle_{\tau}-\frac{1}{3}\right) .
$$

Using $x(\tau)$ and $r(\tau)=R(\tau) / T(\tau)$ we recast the set of three equations $(23,25)$ for $b, R$ and $T$ into a set of two equations for $x$ and $r$. The result reads

$$
\begin{aligned}
\frac{\mathrm{d} r}{\mathrm{~d} \tau}= & B-C\left[1-\frac{x(\tau)}{2}\right] r(\tau)+A r(\tau)-B\left[1-\frac{x(\tau)}{2}\right] r^{2}(\tau) \\
20 \frac{\mathrm{d} x}{\mathrm{~d} \tau}= & -x(\tau)\left\{A^{(1)}+B^{(1)} r(\tau)-20 A-20 C+20 B\left[r(\tau)+\frac{1}{r(\tau)}\right]+20 x(\tau) \frac{C-B r(\tau)}{2}\right\} \\
& -A^{(0)}-B^{(0)} r(\tau)-C^{(0)} r^{-1}(\tau) .
\end{aligned}
$$

Setting the left hand side of Eqs. (28) and (29) to zero one arrives at a set of coupled nonlinear equations for the stationary values $r_{\infty} \equiv r(\tau \rightarrow \infty)$ and $x_{\infty} \equiv x(\tau \rightarrow \infty)$. Instead of solving these equations directly (which has been done for the complete set $(23-25)$ ), we resort to an iteration scheme: At the outset we calculate a first approximation of the temperature ratio $r_{\infty}^{(0)}$ neglecting correlations, that is, for $x=0$. Hence we assume that for moderate inelasticity and roughness the temperature ratio is not noticeably affected by the rotational-translational coupling; this is confirmed below by the direct simulations. The result reads

$$
r_{\infty}^{(0)}=\frac{A-C}{2 B}+\sqrt{1+\frac{(A-C)^{2}}{4 B^{2}}} .
$$

Using this value for the steady-state temperature ratio we then proceed to calculate an approximate value of $x_{\infty}$

$$
x_{\infty}^{(0)}=-\frac{A^{(0)}+B^{(0)} r_{\infty}^{(0)}+C^{(0)} / r_{\infty}^{(0)}}{A^{(1)}+B^{(1)} r_{\infty}^{(0)}-40 C+40 B / r_{\infty}^{(0)}}
$$

where we use the fact, that

$$
B\left[r_{\infty}^{(0)}+1 / r_{\infty}^{(0)}\right]=A-C+2 B / r_{\infty}^{(0)}
$$

and neglect the terms quadratic in $x_{\infty}$ assuming that they are small. The obtained $x_{\infty}$ is indeed small, which (together with the simulation data) confirms a posteriori the validity of the above assumption. In principle, one could further iterate to get better approximations, but we find that the results are reasonably good already at this stage. For the more intuitive variable, $\cos ^{2} \theta$, Eq. (31) implies

$$
\left\langle\cos ^{2} \theta\right\rangle_{\infty} \approx \frac{1}{3}-\frac{6}{5} \frac{A^{(0)}+B^{(0)} r_{\infty}^{(0)}+C^{(0)} / r_{\infty}^{(0)}}{A^{(1)}+B^{(1)} r_{\infty}^{(0)}+40 B / r_{\infty}^{(0)}-40 C}
$$




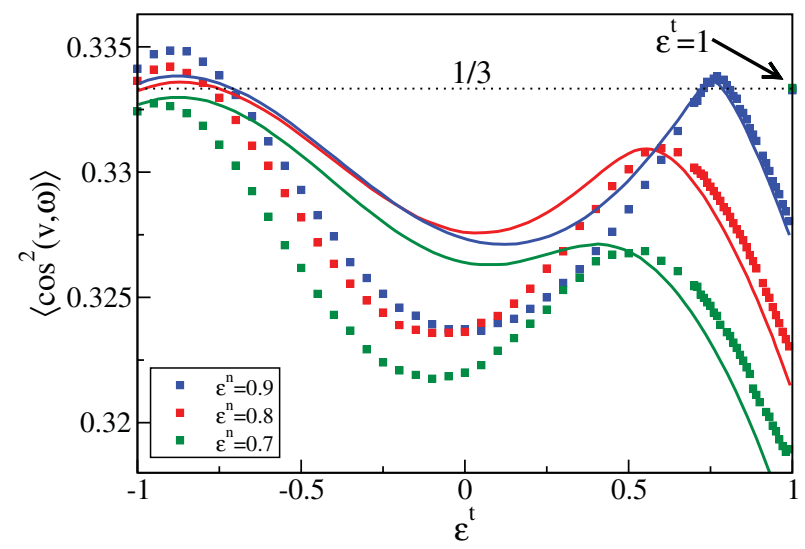

Fig. 1. Steady-state value of $\left\langle\cos ^{2} \theta\right\rangle_{\infty}$ as a function of the coefficient of tangential restitution, $\varepsilon_{t}$, for different $\varepsilon_{n}$. The predictions of the analytical theory, Eq. (33), are depicted by lines and points indicate the simulation data by DSMC. The line of vanishing correlations, $\left\langle\cos ^{2} \theta\right\rangle=1 / 3$ is shown, as well as the isolated point $\varepsilon_{t}=1$, which refers to the system of perfectly smooth hard spheres. Note the existence of non-vanishing correlations even in the limit of smooth spheres, $\varepsilon_{t} \rightarrow 1$ (see Eq. (34)).

Note that the steady-state solution of Eqs. (28), (29) is a stable one, which is discussed in detail below for the particular case of nearly smooth particles.

Figure 1 shows the steady-state value of the correlation factor $\left\langle\cos ^{2} \theta\right\rangle_{\infty}$ as a function of $\varepsilon_{t}$ for different values of $\varepsilon_{n}$ in comparison with DSMC results. Obviously, theory as well as simulations show that both types of correlations may occur, $\left\langle\cos ^{2} \theta\right\rangle<1 / 3$, as for a sliced tennis ball or $\left\langle\cos ^{2} \theta\right\rangle>1 / 3$ as for a rifled bullet. The dependence of the correlations on $\varepsilon_{t}$ is nonmonotonic with the strongest correlations for $\varepsilon_{t} \sim 0$ and $\varepsilon_{t} \rightarrow 1$. Even though the dependence on $\varepsilon_{n}$ is also not strictly monotonic, the dominant tendency is an increase of correlations with decreasing $\varepsilon_{n}$, i.e. increasing inelasticity. The agreement between theory and computer experiment is excellent for small inelasticity. Moreover, even for significant dissipation the theory is able to reproduce qualitatively the simulation results.

Decreasing the moment of inertia, $q$, turns the magnitude of the correlations more sensitive to changes in the coefficients of tangential restitution, as one can see from Figure 2. Interestingly, varying the moment of inertia can even alter the type of the correlations: For instance, for $q=1 / 5$ there exists a region for $\varepsilon_{t}>0$, where the rotation axis is preferably directed along the linear velocity, $\left\langle\cos ^{2} \theta\right\rangle_{\infty}>1 / 3$, while for $q=2 / 3$ there is no such region.

Figure 3 (upper panel) illustrates the analytical result, Eq. (33) for the whole range of parameters $\varepsilon_{t}$ and $\varepsilon_{n}$. Note that for the majority of values of the coefficients, $\left\langle\cos ^{2} \theta\right\rangle_{\infty}<1 / 3$, that is, in most cases the axes tend to be perpendicular to each other. Only in two small regions of the parameter space the axes are preferably parallel. The correlations vanish only for combinations of $\varepsilon_{n}$ and $\varepsilon_{t}$ indicated by full lines. Dashed lines show curves of constant $r$. Strong correlations appear for large deviations from equipartition. This is shown more clearly in the middle and bottom panels of Fig. 3 which demonstrate the rather strong influence of the moment of inertia $I$ on the correlation factor $\left\langle\cos ^{2} \theta\right\rangle_{\infty}$.

To check the assumption that strong correlations occur for strong deviations from equipartition, we plot in Fig. 4 the correlation factor $\left\langle\cos ^{2} \theta\right\rangle_{\infty}$ as a function of $r_{\infty}$ and $\varepsilon_{t}$. Technically this may be done, using $\varepsilon_{n}=\varepsilon_{n}\left(r_{\infty}\right)$-the inverse function of $r_{\infty}=r_{\infty}\left(\varepsilon_{n}\right)$, given by Eq. (30), for each fixed $\varepsilon_{t}$. Note that pronounced correlations are present mainly for strong dissipation and large temperature ratios. Also note the small range of admissible temperature ratios for very rough spheres.

Analyzing Eq. (33) in the limit of vanishing roughness,

$$
K^{(0)} \equiv \lim _{\varepsilon_{t} \rightarrow 1}\left\langle\cos ^{2} \theta\right\rangle_{\infty}-\frac{1}{3}=-\frac{3}{8} \frac{1-\varepsilon_{n}}{7-\varepsilon_{n}}
$$




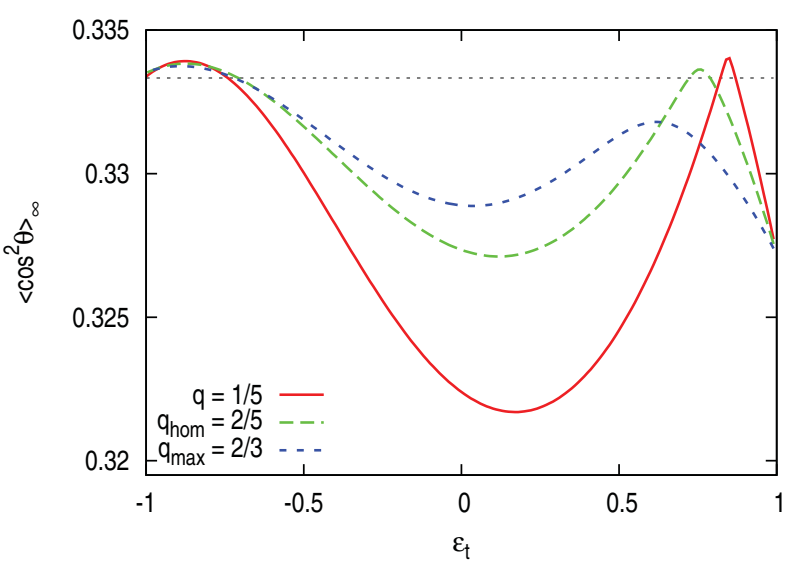

Fig. 2. Steady-state value of $\left\langle\cos ^{2} \theta\right\rangle_{\infty}$ for $\varepsilon_{n}=0.9$ as a function of $\varepsilon_{t}$ and for different moments of inertia of a grain (see also Fig. 7). With the decreasing moment of inertia the correlations become more sensitive to variations of the coefficient of tangential restitution.

we see that even the smallest roughness induces finite correlations, for any given (fixed) value of the coefficient of normal restitution, $\varepsilon_{n} \neq 1$. Physically, this follows from the fact that for a very small roughness the rotation motion dominates in the steady-state. As the result, from the collision rules (3) follows that after a collision, the linear velocity $\mathbf{v}_{1}^{\prime}$ of a particle possesses a component, perpendicular to its angular velocity, which does not vanish upon averaging, in contrast to other contributions to $\mathbf{v}_{1}^{\prime}$ (see [37] for more detailed discussion of this effect). For $\varepsilon_{t}=$ 1 , that is, for perfectly smooth spheres, the initial rotational velocity of the particles is preserved. Therefore, the initial rotational energy is preserved as well and $r$ does not reach a steady state. On the other hand $\left\langle\cos ^{2} \theta\right\rangle$ relaxes to the steady-state value $1 / 3$ once the correlations in the initial values of the translational velocities are lost due to collisions. Hence a straightforward expansion around $\varepsilon_{t}=1$ is problematic, or at least should be done with much care, as long as there is a finite inelasticity $\varepsilon_{n} \neq 1$. [See also the discussion of relaxation times in the following paragraph.]

\subsection{Relaxation to the steady-state}

So far we have discussed the steady-state with a scaling solution, which is characterized by constant $r$ and $\left\langle\cos ^{2} \theta\right\rangle$. It is also of interest to understand, how this steady-state is reachedstarting from arbitrary initial conditions.

Of particular interest is the limit of almost smooth spheres $\eta_{t} \propto \varepsilon_{t}-1 \ll 1$ [see the definition, Eq. (5)]. While the decay of the rotational temperature $R$ and the translational temperature $T$ takes place extremely slowly, that is, with a rate $\sim \eta_{t} \ll 1$ [see Eq. (23) with $r=R / T \simeq A / B$ in this limit], the relaxation of the temperature ratio, $r=R / T$ as well as of the correlation factor $x \sim\left(\left\langle\cos ^{2} \theta\right\rangle-1 / 3\right)$ occurs on the collision time scale. Indeed, in this limit one can write using Eqs. (28), (29) and the definitions of the coefficients (24), (26),

$$
\frac{d r}{d \tau} \simeq-\eta_{n}\left(1-\eta_{n}\right)\left(r / r_{\infty}^{(0)}\right)\left(r-r_{\infty}^{(0)}\right)
$$

with $r_{\infty}^{(0)} \simeq A / B \sim 1 / \eta_{t}^{2} \gg 1$ from Eq. (30). This implies that $r$ relaxes to its steady-state value exponentially fast with a rate $\eta_{n}\left(1-\eta_{n}\right)=\mathcal{O}(1)$ (that is, on the collision time scale), while both temperatures $T$ and $R$ continue to decay with the same small rate.

To analyse the relaxation of $x(\tau)$ to its steady state value, we use Eq. (29) and approximate $r(\tau)$ by its steady state value $r_{\infty}$ :

$$
\frac{\mathrm{d} x}{\mathrm{~d} \tau}=-a_{0}-a_{1} x-a_{2} x^{2}
$$



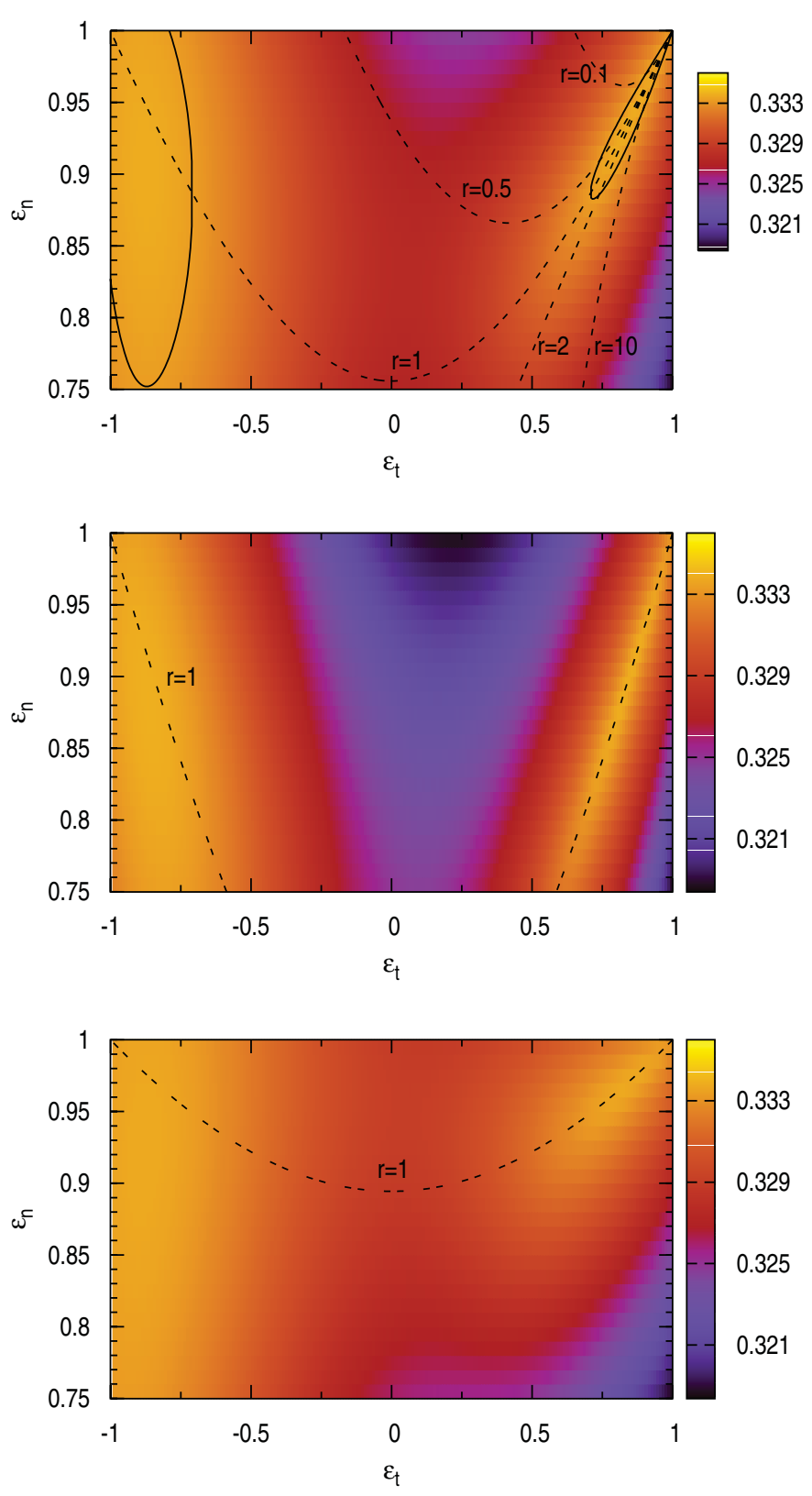

Fig. 3. Steady-state value $\left\langle\cos ^{2} \theta\right\rangle_{\infty}$ (color coded) as a function of normal $\left(\varepsilon_{n}\right)$ and tangential $\left(\varepsilon_{t}\right)$ coefficients of restitution. The stationary value of the temperature ratio $r$ is superimposed through the dashed contour lines. The solid lines indicate vanishing correlations $\left(\left\langle\cos ^{2} \theta\right\rangle_{\infty}=1 / 3\right)$. The moment of inertia $q=2 / 5$ (upper panel) corresponds to homogeneous spheres. The middle and bottom panel show the same data for $q=1 / 5$ and $q=2 / 3$, respectively.

where

$$
\begin{aligned}
& a_{0}=\frac{1}{20}\left[A^{(0)}+B^{(0)} r_{\infty}+C^{(0)} r_{\infty}^{-1}\right] \\
& a_{1}=\frac{1}{20} A^{(1)}+\frac{1}{20} B^{(1)} r_{\infty}-A-C+B\left(r_{\infty}+r_{\infty}^{-1}\right) \\
& a_{2}=\frac{1}{2}\left(C-B r_{\infty}\right) .
\end{aligned}
$$




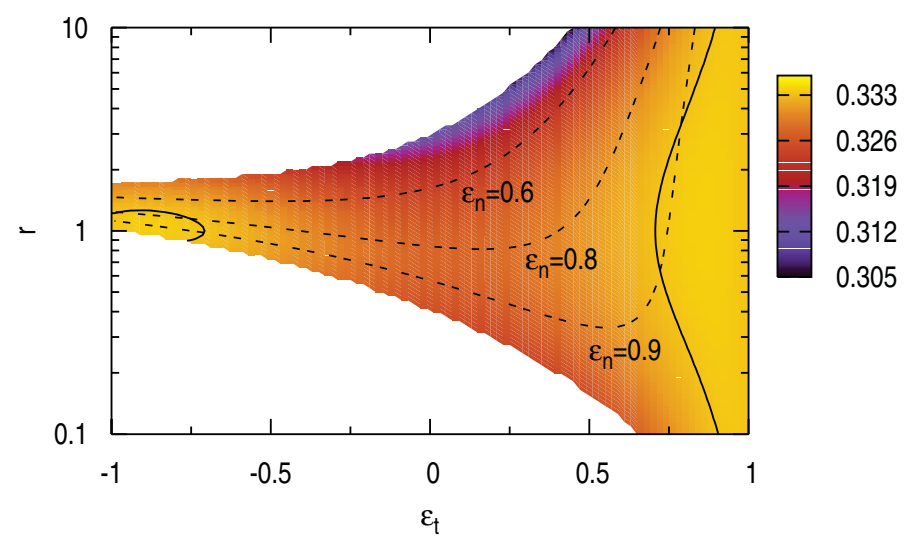

Fig. 4. Steady-state value of $\left\langle\cos ^{2} \theta\right\rangle_{\infty}$ (color coded) as a function of the temperature ratio $r_{\infty}$ and the coefficient of tangential restitution $\varepsilon_{t}$. As previously, the solid lines indicate vanishing correlations and the dashed lines follow constant values of the coefficient of normal restitution $\varepsilon_{n}$. Note the logarithmic scale for the $r$-axis. The ragged border is an artifact of the limited numerical resolution.

The above equation with the initial condition $x(0)=0$ is solved by

$$
x(\tau)-x_{\infty}=-\frac{x_{\infty}}{1-\tanh \phi}\left[1-\tanh \left(\frac{\tau}{\tau_{\text {rel }}}+\phi\right)\right],
$$

with the relaxation time

$$
\tau_{\text {rel }}=\frac{1}{2} \sqrt{a_{1}^{2}-4 a_{0} a_{2}}
$$

and $\tanh \phi=a_{1} / \sqrt{a_{1}^{2}-4 a_{0} a_{2}}$. Evaluating the coefficients for typical values of $\varepsilon_{t}$ and $\varepsilon_{n}$, we find that the relaxation of the correlation factor $\left\langle\cos ^{2} \theta\right\rangle_{t}$ to its steady-state also occurs within a few collisions per particle. This is illustrated in Fig. 5 , where we plot the relaxation time $\tau_{\text {rel }}$ given by Eq. (39).

We wish to stress here again, that the relaxation on the collisional time scale to the steady state values applies only to the temperature ratio and the mean square cosine of the angle between linear and angular velocity. For nearly smooth particles, $\varepsilon_{t} \rightarrow 1$, the relaxation of the rotational and translational temperatures is, nevertheless, a very slow process, which proceeds with a small rate, tending to zero as $\varepsilon_{t} \rightarrow 1$.

To demonstrate the existence of several time regimes we discuss in the following an instructive example. We initialize the particles with $\boldsymbol{\omega}=0$ corresponding to $r=0$. The collision parameters are $\varepsilon_{n}=\varepsilon_{t}=0.8$ so that the asymptotic value of the ratio of temperatures is $r_{\infty}>1$. We expect $r$ to monotonically increase as a function of time - and this is indeed observed as shown in Fig. 6. Now, we can check our hypothesis that correlations are small for values of $r$ close to equipartition. If the hypothesis is correct, we should observe non-monotonic behavior of $\left\langle\cos ^{2} \theta\right\rangle_{t}$. For short times the correlations should be large and of tennis ball type, because grazing collisions are the most effective for spinless particles to gain angular momentum. At intermediate times, when $r \sim 1$, the correlations should be very small or vanishing. In the asymptotic state with $r_{\infty}>1$, one should again observe finite correlations.

These three time regimes are clearly borne out in the time dependent correlations, shown in Fig. 6: (a) In the short time regime $\left(0<t<10^{3}\right)$ correlations are strong and $0<r<1$. (b) At intermediate times $\left(10^{3}<t<10^{5}\right)$ equipartition holds approximately $r \approx 1$ and correlations are small or vanishing. (c) The steady state $\left(t>10^{6}\right)$ is characterized by $r \gg 1$ and finite $\left\langle\cos ^{2} \theta\right\rangle_{\infty}<1 / 3$. The agreement between analytical theory and molecular dynamics is good also for the time-dependent quantities. 


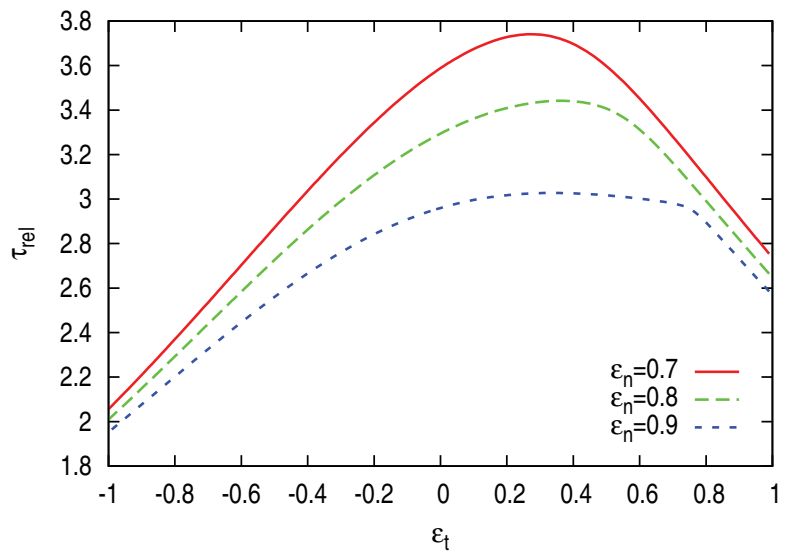

Fig. 5. Relaxation time $\tau_{\text {rel }}$ (in the collision units) of $\left\langle\cos ^{2} \theta\right\rangle_{\tau}$ when it approaches the steady-state value $\left\langle\cos ^{2} \theta\right\rangle_{\infty}$. Note the narrow range of possible values for $\tau_{\text {rel }}$.

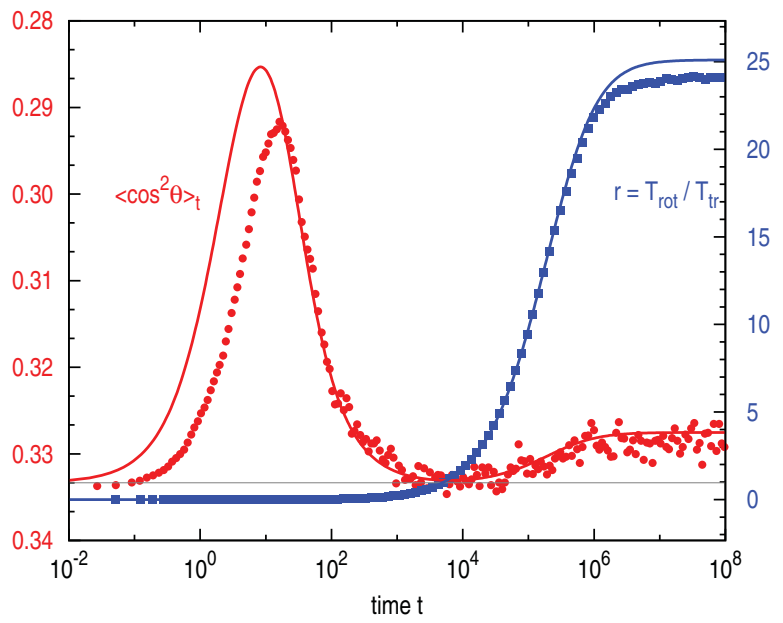

Fig. 6. Relaxation of $\left\langle\cos ^{2} \theta\right\rangle_{t}$ and of the ratio of temperatures $r(t)=R(t) / T(t)$ to the steady state. Dots: molecular dynamics data for 8000 particles, lines: analytical theory. To show that vanishing correlations $\left\langle\cos ^{2} \theta\right\rangle_{t}$ coincide with equipartition, we have chosen the vertical axes, such that the point $r=1$ on the right axis (blue) and the point $\left\langle\cos ^{2} \theta\right\rangle_{t}=1 / 3$ on the left axis (red) have the same vertical height as indicated by a horizontal line.

Figure 7 demonstrates that the moment of inertia of the particles does not change the evolution of $\left\langle\cos ^{2} \theta\right\rangle_{t}$ qualitatively. For the particular choice of the coefficients of restitution the correlations are more pronounced for larger $q=I / m a^{2}$ and fade with decreasing $q$. This however is not a general rule; depending on the coefficients $\varepsilon_{n}$ and $\varepsilon_{t}$, this tendency may reverse.

The correlations between translational and rotational motion also have a noticeable, albeit small impact on the basic characteristics of granular gases - the translational and rotational temperatures. In Fig. 8 we present the time dependence of $R(t) / R^{(0)}(t)$ - the ratio of the rotational temperature $R(t)$ with correlations to the corresponding value $R^{(0)}(t)$ without correlations. The respective ratio $T(t) / T^{(0)}(t)$ for the translational temperature is also plotted. Here we choose the case of large $r \simeq 24\left(\varepsilon_{n}=\varepsilon_{t}=0.8\right)$, which correspond to $\left\langle\cos ^{2} \theta\right\rangle_{\infty}<1 / 3$, that is, for preferably perpendicular rotational and translational velocity. Figure 8 demonstrates that the effect of the correlations on the granular temperatures $R(t)$ and $T(t)$ is indeed small. The corresponding quantity $r(t)=R(t) / T(t)$ is also not sensitive to these correlations. Moreover 


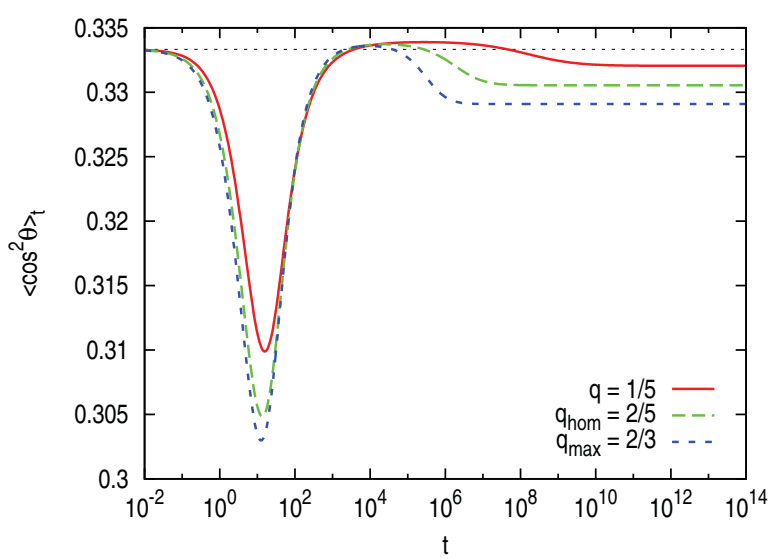

Fig. 7. Impact of the grains' moments of inertia on the evolution and steady-state of $\left\langle\cos ^{2} \theta\right\rangle_{t}$. The system parameters are $\varepsilon_{n}=0.9, \varepsilon_{t}=0.9, r(0)=0.001$ and vanishing initial correlations. The values of $q\left(I=q m a^{2}\right)$ represent spheres with the mass concentrated towards the center $(q=1 / 5)$, the homogeneous spheres $(q=2 / 5)$ and spheres with the mass concentrated mainly in the outer shell $(q=2 / 3)$.

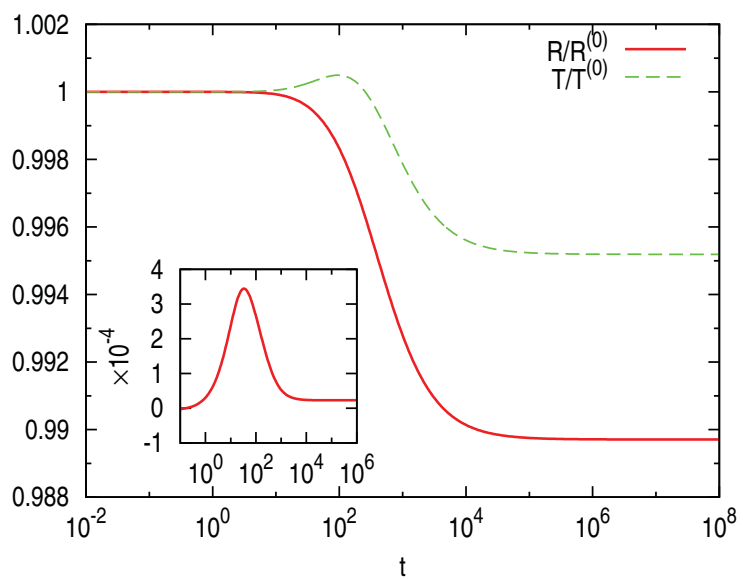

Fig. 8. The ratio of the temperatures calculated with $b(t)$ according to Eq. $(25)$ to those with $b(t) \equiv 0$. The coefficients of restitution are $\varepsilon_{n}=\varepsilon_{t}=0.8$, and the initial ratio of rotational to translational temperatures was set to the steady-state value $r(0)=r_{\infty}$. The inset shows $r(t) / r(0)-1$ as a function of time. Note that the deviation of $r(t)$ from $r_{\infty}$ is always very small.

$r(t)$ does not deviate noticeably from its steady-state value throughout the system's evolution, that is, $\left|r(\tau) / r_{\infty}-1\right| \ll 1$, as shown in the inset of Fig. 8 .

\subsection{Beyond the second moment}

A complete one-particle picture includes the distribution

$$
\mathcal{W}(\cos \theta, v, \omega)=\frac{1}{N} \sum_{i=1}^{N} \delta\left(\cos \theta-\cos \theta_{i}\right) \delta\left(v-v_{i}\right) \delta\left(\omega-\omega_{i}\right) .
$$

Since correlations are developed in collisions, one intuitively expects that particles with larger velocities, that suffer stronger collisions, would show more pronounced orientational correlations; we study these effects by binning the particles velocities. 


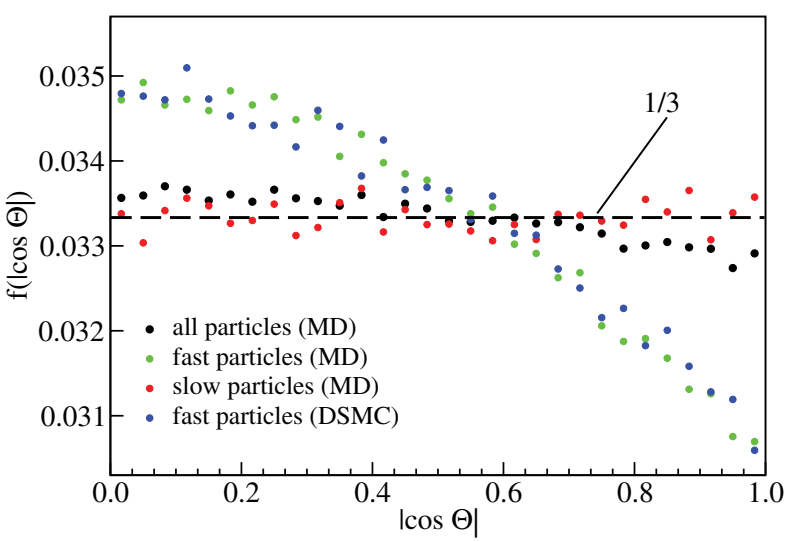

Fig. 9. The angular distribution $f(|\cos \theta|)$ for the system of rough spheres with $\varepsilon_{t}=0.9, \varepsilon_{n}=0.9$ and $q=2 / 5$ in the steady-state. Note that while there is no preferable angle between $\mathbf{v}$ and $\boldsymbol{\omega}$ for slow particles, correlations are clearly visible for fast particles favouring perpendicular linear and angular velocities.

So far we discussed the correlation factor $\left\langle\cos ^{2} \theta\right\rangle_{t}$, which is a second moment of the distribution function $\mathcal{W}(\cos \theta, v, \omega)$. Let us now analyze the distribution function itself. Due to the limited statistics of our numerical data we discriminate only between two classes of particles: the class of fast particles comprising those particles whose linear velocity belongs to the set of the $1 / 3$ largest values and whose angular velocity belongs to the set of the $1 / 3$ largest values. The class of slow particles is defined correspondingly as the set of particles whose linear velocity belongs to the set of the $1 / 3$ smallest values and the angular velocity belongs to the set of the $1 / 3$ smallest values. In Fig. 9 we show the distributions $f(|\cos \theta|)$ for the two classes in comparison with the distribution for all particles using both methods, MD and DSMC. In both cases we skipped the first 20 collisions per particle such that the ratio of temperatures, $r$, has reached its steady-state value. For the MD simulation we used a system of $N=10^{5}$ particles at low density (filling factor $<1 \%$. Then we averaged over 200 snapshots in distance of 1 collision per particle. In case of DSMC we used a system of $N=10^{7}$ particles and made the statistics based on a single snapshot. Both results agree very well. The angular distribution is almost flat for slow particles and cannot be distinguished from the distribution of all particles (within statistical accuracy). On the other hand the fast particles exhibit a nonuniform distribution with a maximum around $\cos \theta=0$. Physically this means that the angle $\theta$ between $\mathbf{v}$ and $\boldsymbol{\omega}$ for slow particles is uniformly distributed within the interval $(0, \pi)$, while for fast particles it lies preferentially around $\theta=\pi / 2$. In other words, for the particular choice of $\varepsilon_{t}=0.9$ and $\varepsilon_{n}=0.9$ the fast particles tend to behave like sliced tennis balls, with $\boldsymbol{\omega}$ perpendicular to $\mathbf{v}$.

\section{Conclusions and outlook}

We have analysed in detail the correlations between rotational and translational motion in a granular gas of frictional particles. Under the assumption of molecular chaos and homogeneity we have developed an analytical theory which accounts for the correlations $\left\langle\cos ^{2} \theta\right\rangle_{t}$ in addition to the rotational $R(t)$ and translational $T(t)$ temperature. We have also performed large scale DSMC simulations as well as event driven simulations to study the evolution of a gas of rough spheres and in particular the above correlations.

We observe that the gas of rough particles always relaxes to a steady-state with constant correlation $\left\langle\cos ^{2} \theta\right\rangle_{\infty}$ and constant ratio $r_{\infty}=R(t) / T(t)$. While the relaxation of $\left\langle\cos ^{2} \theta\right\rangle$ and $r$ to their steady-state values happens on the collisional time scale, the evolution of the rotational and translational temperature in the near-smooth limit $\varepsilon_{t} \rightarrow 1$ is a slow process with a vanishingly small rate $\sim \eta_{t} \sim\left(1-\varepsilon_{t}\right) \ll 1$. Physically, this may be explained as follows. In the near-smooth limit the coupling of the rotational modes to the translational ones becomes very 
weak. The energy of the rotational motion of the particles is almost conserved in collisions and the exchange of energy between the translational and rotational degrees of freedom becomes very slow. Consequently the rotational temperature as well as the translational temperature have a slowly decaying component, governed by this weak exchange of energies. However both temperatures decay with the same slow timescale so that their ratio, $r$, is stationary - after it has reached its steady state on the fast time scale of a few collisions. Simultaneously, $\left\langle\cos ^{2} \theta\right\rangle_{t}$ relaxes to its steady state with a similar rate of the order of a few collisions. We conclude that the relaxation of the temperature ratio, $r$, and the angular correlations is rapid, - independent of the strength of the coupling $\left(1-\varepsilon_{t}\right)$ as long as it is finite. Furthermore the correlations persist up to a vanishingly small roughness and are absent only for perfectly smooth particles, $\varepsilon_{t}=1$; this requires a special care for the expansions around the smooth limit.

Our main results concern the correlation between the directions of rotational and translational velocity in the steady-state: The correlations depend sensitively on the values of the coefficients of restitution and the moment of inertia; for most of the system parameters $\left\langle\cos ^{2} \theta\right\rangle<1 / 3$, implying that linear and angular velocities are preferably orthogonal, like in a sliced tennis ball. Only for a small part of the parameter space $\left\langle\cos ^{2} \theta\right\rangle>1 / 3$, which means that $\mathbf{v}$ and $\boldsymbol{\omega}$ are preferably parallel like in a rifled bullet; the manifold of vanishing correlations (in $\varepsilon_{n}, \varepsilon_{t}$ space) has seemingly zero measure. The correlations are more pronounced for strong deviations from equipartition.

Our approach can be extended in several directions. In the simulations it is straightforward to use more advanced models for the coefficients of restitution as functions of the impact velocity, e.g. [38-41]. It would also be of interest to study the full one-particle distribution. Our results already indicate that more energetic particles have stronger correlations, but a systematic study has yet to be done. Furthermore, one expects to observe correlations not only in very dilute gases, but also in rapidly moving denser systems. Our approximate analytical theory is based on the assumption of homogeneity and the density only enters into the Enskog collision frequency, which sets the time scale. Hence our results for the steady-state are independent of the density. This cannot hold true in a rapidly moving dense system, yet we expect to observe correlations as well. These could be analysed in a molecular dynamics simulation either for a driven [48] or undriven system. Finally, the observed correlations may have important consequences for the stability theory of dilute granular flows: they possibly alter the domain of stability of granular system with respect to shear fluctuations - the main instability of granular flows of smooth particles.

We thank Isaac Goldhirsch for interesting discussions; TK and AZ thank Timo Aspelmeier for help with the MD simulations; TP acknowledges support by a grant from G.I.F., the German-Israeli Foundation for Scientific Research and Development.

\section{Appendix}

\section{A Correlation factor}

We present the details of the analytical calculations, leading to the three self-consistent equations (23) and (25) for $T(t), R(t)$ and $b(t)$. First, we note that he computation of $b(t)$ or $\left\langle\cos ^{2} \theta\right\rangle_{t}$ is severely hampered by the denominator in Eq. (9). Fortunately one can carry out the calculations with the auxiliary variable

$$
\langle\Delta\rangle_{t} \equiv \frac{2}{3 N} \sum_{i=1}^{N} \mathbf{v}_{i}^{2} \boldsymbol{\omega}_{i}^{2} P_{2}\left(\cos \theta_{i}\right) .
$$

Its relation to our set of dynamical variables can be established by essentially the same steps as leading from Eq. (20) to Eq. (22):

$$
30 b(t) \frac{T(t) R(t)}{q m^{2} a^{2}}=\langle\Delta\rangle_{t} \frac{q m^{2} a^{2}}{T(t) R(t)} .
$$


In the case of vanishing correlations we have $\langle\Delta\rangle_{t}=0$. Positive (negative) values correspond to a preference of a parallel (perpendicular) orientation.

Owing to the assumptions of spatial homogeneity and molecular chaos it suffices to consider the phase space of only a single pair of particles (without loss of generality these shall be labeled 1 and 2). Integrating out the spatial degrees of freedom and using the definition of the pair correlation function

$$
N(N-1) \int \mathrm{d} \mathbf{r}_{3} \ldots \mathrm{d} \mathbf{r}_{N} g_{N}\left(\mathbf{r}_{1}, \ldots \mathbf{r}_{N}\right)=n^{2} g_{2}\left(r_{12}\right),
$$

with $n$ being the number density of the gas (e.g. [6]) we obtain

$$
\begin{aligned}
\left\langle i \mathcal{L}_{+} \Delta\right\rangle_{t}= & \nu N \int_{v_{1}} \int_{v_{2}} \int_{\omega_{1}} \int_{\omega_{2}}\left(\hat{\mathbf{n}} \cdot \mathbf{v}_{12}\right) \Theta\left(-\hat{\mathbf{n}} \cdot \mathbf{v}_{12}\right) \\
& \times\left[1+b(t) \mathbf{v}_{1}^{2} \boldsymbol{\omega}_{1}^{2} P_{2}\left(\cos \theta_{1}\right)\right]\left[1+b(t) \mathbf{v}_{2}^{2} \boldsymbol{\omega}_{2}^{2} P_{2}\left(\cos \theta_{2}\right)\right]\left(\hat{b}_{12}-1\right) \Delta
\end{aligned}
$$

where $\hat{\mathbf{n}}$ is an arbitrary but fixed unit vector, $\nu=-8 \pi n a^{2} g_{2}(2 a)$ and we used the shorthand notations

$$
\int_{v_{i}} \equiv\left(\frac{m}{2 \pi T}\right)^{3 / 2} \int \mathrm{d}^{3} v_{i} \exp \left(-\frac{m \mathbf{v}_{i}^{2}}{2 T}\right) \quad \text { and } \quad \int_{\omega_{i}} \equiv\left(\frac{I}{2 \pi R}\right)^{3 / 2} \int \mathrm{d}^{3} \omega_{i} \exp \left(-\frac{I \boldsymbol{\omega}_{i}^{2}}{2 R}\right) .
$$

In the following we will drop the $b^{2}(t)$-term stemming from the product of the two one particle distribution functions $f$, assuming that it is small. This assumption is confirmed a posteriori analytically, as well as in the numerical simulations. Hence, in what follows we consider only terms first order in $b(t)$.

The calculation of $\left(\hat{b}_{12}-1\right) \Delta$ is obviously rather involved and, thus, it needs to be broken up to stay tractable. It is convenient to introduce relative integration variables

$$
\mathbf{v} \equiv \mathbf{v}_{12} / \sqrt{2}, \quad \mathbf{V} \equiv\left(\mathbf{v}_{1}+\mathbf{v}_{2}\right) / \sqrt{2}, \quad \boldsymbol{\omega} \equiv \boldsymbol{\omega}_{12} / \sqrt{2}, \quad \boldsymbol{\Omega} \equiv\left(\boldsymbol{\omega}_{1}+\boldsymbol{\omega}_{2}\right) / \sqrt{2} .
$$

The term $\left\langle i \mathcal{L}_{+} \Delta\right\rangle_{t}$ can be broken up along two different principles. First, one can make the dependence on $b(t)$ explicit, that is,

$$
\left\langle i \mathcal{L}_{+} \Delta\right\rangle_{t}=\left\langle\left(\hat{b}_{12}-1\right) \Delta\right\rangle^{(0)}+b(t)\left\langle\left(\hat{b}_{12}-1\right) \Delta\right\rangle^{(1)}+\mathcal{O}\left(b^{2}\right)
$$

where for any function $F$

$$
\begin{aligned}
& \langle F\rangle^{(0)}=\nu \int_{v_{1}} \int_{v_{2}} \int_{\omega_{1}} \int_{\omega_{2}}\left(\hat{\mathbf{n}} \cdot \mathbf{v}_{12}\right) \Theta\left(-\hat{\mathbf{n}} \cdot \mathbf{v}_{12}\right) F \\
& \langle F\rangle^{(1)}=\nu \int_{v_{1}} \int_{v_{2}} \int_{\omega_{1}} \int_{\omega_{2}}\left(\hat{\mathbf{n}} \cdot \mathbf{v}_{12}\right) \Theta\left(-\hat{\mathbf{n}} \cdot \mathbf{v}_{12}\right)\left[\mathbf{v}_{1}^{2} \boldsymbol{\omega}_{1}^{2} P_{2}\left(\cos \theta_{1}\right)+\mathbf{v}_{2}^{2} \boldsymbol{\omega}_{2}^{2} P_{2}\left(\cos \theta_{2}\right)\right] F .
\end{aligned}
$$

In order to be able to exploit some further symmetries it is advisable to split up the last average again,

$$
\langle F\rangle^{(1)}=\langle F\rangle^{\text {even }}+\langle F\rangle^{\text {odd }}
$$

where

$$
\begin{aligned}
\langle F\rangle^{\text {even }}= & \frac{3 \sqrt{2}}{4} \nu \int_{v} \int_{V} \int_{\omega} \int_{\Omega}(\hat{\mathbf{n}} \cdot \mathbf{v}) \Theta(-\hat{\mathbf{n}} \cdot \mathbf{v}) \\
& \times\left[(\mathbf{V} \cdot \boldsymbol{\Omega})^{2}+(\mathbf{V} \cdot \boldsymbol{\omega})^{2}+(\mathbf{v} \cdot \boldsymbol{\Omega})^{2}+(\mathbf{v} \cdot \boldsymbol{\omega})^{2}-\frac{1}{3}\left(\mathbf{V}^{2}+\mathbf{v}^{2}\right)\left(\boldsymbol{\Omega}^{2}+\boldsymbol{\omega}^{2}\right)\right] F
\end{aligned}
$$


involves only even powers of $\mathbf{V}, \boldsymbol{\omega}, \boldsymbol{\Omega}$ and

$$
\begin{aligned}
\langle F\rangle^{\text {odd }}= & \frac{3 \sqrt{2}}{2} \nu \int_{v} \int_{V} \int_{\omega} \int_{\Omega}(\hat{\mathbf{n}} \cdot \mathbf{v}) \Theta(-\hat{\mathbf{n}} \cdot \mathbf{v}) \\
& \times\left[(\mathbf{V} \cdot \boldsymbol{\Omega})(\mathbf{v} \cdot \boldsymbol{\omega})+(\mathbf{V} \cdot \boldsymbol{\omega})(\mathbf{v} \cdot \boldsymbol{\Omega})-\frac{2}{3}(\mathbf{V} \cdot \mathbf{v})(\boldsymbol{\Omega} \cdot \boldsymbol{\omega})\right] F
\end{aligned}
$$

in contrast involves only the odd powers of these quantities. Independently we can write

$$
\Delta=\Delta_{A}-\Delta_{B} / 3
$$

where

$$
\Delta_{A} \equiv \sum_{i}\left(\mathbf{v}_{i} \cdot \boldsymbol{\omega}_{i}\right)^{2} \quad \text { and } \quad \Delta_{B} \equiv \sum_{i} \mathbf{v}_{i}^{2} \boldsymbol{\omega}_{i}^{2} .
$$

First we address the $\Delta_{A}$-part. Applying the collision rule to $\Delta_{A}$ yields

$$
\begin{aligned}
\left(\hat{b}_{12}-1\right) \Delta_{A}=(\boldsymbol{\delta} \cdot \boldsymbol{\omega})^{2}+(\boldsymbol{\delta} \cdot \boldsymbol{\Omega})^{2}+\frac{1}{q^{2} a^{2}}[(\hat{\mathbf{n}} \times \boldsymbol{\delta}) \cdot \mathbf{v}]^{2}+\frac{1}{q^{2} a^{2}}[(\hat{\mathbf{n}} \times \boldsymbol{\delta}) \cdot \mathbf{V}]^{2}-\sqrt{2}(\boldsymbol{\delta} \cdot \boldsymbol{\Omega})(\mathbf{v} \cdot \boldsymbol{\Omega}) \\
\quad-\sqrt{2}(\boldsymbol{\delta} \cdot \boldsymbol{\Omega})(\mathbf{V} \cdot \boldsymbol{\omega})-\sqrt{2}(\boldsymbol{\delta} \cdot \boldsymbol{\omega})(\mathbf{v} \cdot \boldsymbol{\omega})-\sqrt{2}(\boldsymbol{\delta} \cdot \boldsymbol{\omega})(\mathbf{V} \cdot \boldsymbol{\Omega})+\frac{\sqrt{2}}{q a}(\mathbf{v} \cdot \boldsymbol{\Omega})(\hat{\mathbf{n}} \times \boldsymbol{\delta}) \cdot \mathbf{v} \\
\quad+\frac{\sqrt{2}}{q a}(\mathbf{V} \cdot \boldsymbol{\omega})(\hat{\mathbf{n}} \times \boldsymbol{\delta}) \cdot \mathbf{v}+\frac{\sqrt{2}}{q a}(\mathbf{v} \cdot \boldsymbol{\omega})(\hat{\mathbf{n}} \times \boldsymbol{\delta}) \cdot \mathbf{V}+\frac{\sqrt{2}}{q a}(\mathbf{V} \cdot \boldsymbol{\Omega})(\hat{\mathbf{n}} \times \boldsymbol{\delta}) \cdot \mathbf{V} \\
\quad-\frac{2}{q a}(\boldsymbol{\delta} \cdot \boldsymbol{\Omega})(\hat{\mathbf{n}} \times \boldsymbol{\delta}) \cdot \mathbf{v}-\frac{2}{q a}(\boldsymbol{\delta} \cdot \boldsymbol{\omega})(\hat{\mathbf{n}} \times \boldsymbol{\delta}) \cdot \mathbf{V}
\end{aligned}
$$

and invoking the definition of $\boldsymbol{\delta}$, Eq. (4), we obtain

$$
\begin{aligned}
\left\langle\left(\hat{b}_{12}\right.\right. & \left.-1) \Delta_{A}\right\rangle^{(0)} \\
= & 2\left(2 \eta_{t}^{2}-2 \eta_{t}+\frac{\eta_{t}^{2}}{q^{2}}-\frac{\eta_{t}}{q}+\frac{2 \eta_{t}^{2}}{q}\right)\left\langle(\mathbf{v} \cdot \boldsymbol{\omega})^{2}\right\rangle^{(0)}+2 \frac{\eta_{t}}{q}\left(\frac{\eta_{t}}{q}-1\right)\left\langle(\boldsymbol{\omega} \cdot \mathbf{V})^{2}\right\rangle^{(0)} \\
& +2\left[2\left(\eta_{n}-\eta_{t}\right)^{2}+\frac{\eta_{t}^{2}}{q^{2}}-\frac{2 \eta_{t}}{q}\left(\eta_{n}-\eta_{t}\right)\right]\left\langle(\hat{\mathbf{n}} \cdot \mathbf{v})^{2}(\hat{\mathbf{n}} \cdot \boldsymbol{\omega})^{2}\right\rangle^{(0)} \\
& +2 \frac{\eta_{t}^{2}}{q^{2}}\left\langle(\hat{\mathbf{n}} \cdot \mathbf{V})^{2}(\hat{\mathbf{n}} \cdot \boldsymbol{\omega})^{2}\right\rangle^{(0)}+2 \eta_{t}^{2} a^{2}\left\langle[(\hat{\mathbf{n}} \times \mathbf{\Omega}) \cdot \boldsymbol{\omega}]^{2}\right\rangle^{(0)}+2 \frac{\eta_{t}^{2}}{q^{2} a^{2}}\left\langle[(\hat{\mathbf{n}} \times \mathbf{v}) \cdot \mathbf{V}]^{2}\right\rangle^{(0)} \\
& +4\left[\left(2 \eta_{t}-1\right)\left(\eta_{n}-\eta_{t}\right)-\frac{\eta_{t}^{2}}{q^{2}}+\frac{1}{2} \frac{\eta_{t}}{q}-\frac{\eta_{t}^{2}}{q}+\frac{\eta_{t}}{q}\left(\eta_{n}-\eta_{t}\right)\right]\langle(\hat{\mathbf{n}} \cdot \mathbf{v})(\hat{\mathbf{n}} \cdot \boldsymbol{\omega})(\mathbf{v} \cdot \boldsymbol{\omega})\rangle^{(0)} \\
& -\frac{2 \eta_{t}}{q}\left(\frac{2 \eta_{t}}{q}-1\right)\langle(\hat{\mathbf{n}} \cdot \mathbf{V})(\hat{\mathbf{n}} \cdot \boldsymbol{\omega})(\mathbf{V} \cdot \boldsymbol{\omega})\rangle^{(0)}
\end{aligned}
$$

The terms that vanish by symmetry are already left out at this point. The contributions to $\left\langle\left(\hat{b}_{12}-1\right) \Delta_{A}\right\rangle^{\text {even }}$ have exactly the same form. For $\left\langle\left(\hat{b}_{12}-1\right) \Delta_{A}\right\rangle$ odd one finds the following 
contributions

$$
\begin{aligned}
& \left\langle\left(\hat{b}_{12}-1\right) \Delta_{A}\right\rangle^{\text {odd }} \\
& =\left(\frac{4 \eta_{t}^{2}}{q}-\frac{4 \eta_{t}}{q}-4 \eta_{t}\right)\langle(\mathbf{v} \cdot \boldsymbol{\omega})(\mathbf{V} \cdot \boldsymbol{\Omega})\rangle^{\text {odd }}-\frac{4 \eta_{t}}{q}\left(\eta_{n}-\eta_{t}\right)\langle(\hat{\mathbf{n}} \cdot \mathbf{v})(\hat{\mathbf{n}} \cdot \mathbf{V})(\hat{\mathbf{n}} \cdot \boldsymbol{\omega})(\hat{\mathbf{n}} \cdot \boldsymbol{\Omega})\rangle^{\text {odd }} \\
& \quad+\frac{2 \eta_{t}}{q}\left(1-2 \eta_{t}\right)\langle(\hat{\mathbf{n}} \cdot \mathbf{V})(\hat{\mathbf{n}} \cdot \boldsymbol{\Omega})(\mathbf{v} \cdot \boldsymbol{\omega})\rangle^{\text {odd }}-\frac{4 \eta_{t}^{2}}{q}\langle[(\hat{\mathbf{n}} \times \mathbf{v}) \cdot \mathbf{V}][(\hat{\mathbf{n}} \times \boldsymbol{\Omega}) \cdot \boldsymbol{\omega}]\rangle^{\text {odd }} \\
& \quad+\left[4\left(\frac{\eta_{t}}{q}-1\right)\left(\eta_{n}-\eta_{t}\right)+\frac{2 \eta_{t}}{q}\right]\langle(\hat{\mathbf{n}} \cdot \mathbf{v})(\hat{\mathbf{n}} \cdot \boldsymbol{\omega})(\mathbf{V} \cdot \boldsymbol{\Omega})\rangle^{\text {odd }} .
\end{aligned}
$$

Correspondingly, the $\Delta_{B}$-part may be written as

$$
\begin{aligned}
\left\langle\left(\hat{b}_{12}-1\right) \Delta_{B}\right\rangle^{(0)} & \\
= & \frac{2 \eta_{t}}{q}\left(\frac{\eta_{t}}{q}-1\right)\left(2 \eta_{t}-1\right)^{2}\left\langle\mathbf{v}^{2}(\mathbf{n} \times \boldsymbol{\Omega})^{2}\right\rangle^{(0)}+\frac{2 \eta_{t}}{q}\left(\frac{\eta_{t}}{q}-1\right)\left\langle\mathbf{V}^{2}(\mathbf{n} \times \boldsymbol{\Omega})^{2}\right\rangle^{(0)} \\
& +\frac{2 \eta_{t}^{2}}{q^{2} a^{2}}\left(2 \eta_{t}-1\right)^{2}\left\langle\mathbf{v}^{2}(\hat{\mathbf{n}} \times \mathbf{v})^{2}\right\rangle^{(0)}+\frac{2 \eta_{t}^{2}}{q^{2} a^{2}}\left\langle\mathbf{V}^{2}(\hat{\mathbf{n}} \times \mathbf{v})^{2}\right\rangle^{(0)}+4 \eta_{t}\left(\eta_{t}-1\right)\left\langle\mathbf{v}^{2} \boldsymbol{\omega}^{2}\right\rangle^{(0)} \\
& +4\left(\eta_{n}^{2}-\eta_{n}-\eta_{t}^{2}+\eta_{t}\right)\left\langle(\hat{\mathbf{n}} \cdot \mathbf{v})^{2} \boldsymbol{\omega}^{2}\right\rangle^{(0)} \\
& +\frac{8 \eta_{t}}{q}\left(\frac{\eta_{t}}{q}-1\right)\left(\eta_{n}^{2}-\eta_{n}-\eta_{t}^{2}+\eta_{t}\right)\left\langle(\hat{\mathbf{n}} \cdot \mathbf{v})^{2}(\hat{\mathbf{n}} \times \boldsymbol{\Omega})^{2}\right\rangle^{(0)} \\
& +\frac{8 \eta_{t}^{2}}{q}\left(\frac{2 \eta_{t}}{q}-1\right)\left(2 \eta_{t}-1\right)\left\langle[(\hat{\mathbf{n}} \times \mathbf{v}) \cdot \boldsymbol{\Omega}]^{2}\right\rangle^{(0)} \\
& +\frac{8 \eta_{t}^{2}}{q^{2} a^{2}}\left(\eta_{n}^{2}-\eta_{n}-\eta_{t}^{2}+\eta_{t}\right)\left\langle(\hat{\mathbf{n}} \cdot \mathbf{v})^{2}(\hat{\mathbf{n}} \times \mathbf{v})^{2}\right\rangle^{(0)} \\
& +2 \eta_{t}^{2} a^{2}\left\langle(\hat{\mathbf{n}} \times \boldsymbol{\Omega})^{2} \boldsymbol{\omega}^{2}\right\rangle^{(0)}+2 \eta_{t}^{2} a^{2}\left\langle(\hat{\mathbf{n}} \times \boldsymbol{\Omega})^{2} \boldsymbol{\Omega}^{2}\right\rangle^{(0)} \\
& +\frac{8 \eta_{t}^{3}}{q} a^{2}\left(\frac{\eta_{t}}{q}-1\right)\left\langle(\hat{\mathbf{n}} \times \boldsymbol{\Omega})^{4}\right\rangle^{(0)}+\frac{8 \eta_{t}^{4}}{q^{2}}\left\langle(\hat{\mathbf{n}} \times \boldsymbol{\Omega})^{2}(\hat{\mathbf{n}} \times \mathbf{v})^{2}\right\rangle^{(0)}
\end{aligned}
$$

The contributions to $\left\langle\left(\hat{b}_{12}-1\right) \Delta_{B}\right\rangle^{\text {even }}$ again are formally equivalent to the above expression. This leaves us with

$$
\begin{aligned}
& \left\langle\left(\hat{b}_{12}-1\right) \Delta_{B}\right\rangle^{\text {odd }} \\
& =-\frac{4 \eta_{t}}{q}\left(2 \eta_{t}-1\right)\langle(\hat{\mathbf{n}} \cdot \boldsymbol{\omega})(\hat{\mathbf{n}} \cdot \boldsymbol{\Omega})(\mathbf{v} \cdot \mathbf{V})\rangle^{\text {odd }}+\frac{8 \eta_{t}^{2}}{q}\langle[(\hat{\mathbf{n}} \times \mathbf{v}) \cdot \boldsymbol{\omega}][(\hat{\mathbf{n}} \times \boldsymbol{\Omega}) \cdot \mathbf{V}]\rangle^{\text {odd }} \\
& \quad-\frac{8 \eta_{t}}{q}\left(\eta_{n}-\eta_{t}\right)\langle(\hat{\mathbf{n}} \cdot \mathbf{v})(\hat{\mathbf{n}} \cdot \mathbf{V})(\hat{\mathbf{n}} \cdot \boldsymbol{\omega})(\hat{\mathbf{n}} \cdot \mathbf{\Omega})\rangle^{\text {odd }} .
\end{aligned}
$$

We have now reduced the problem to the tedious but straightforward calculation of a considerable number of averages. This task is best suited for a computer algebra system and thus we only tabulate the results. To simplify the notation we introduce the abbreviations $\tilde{\nu} \equiv \nu \sqrt{T / m \pi}$, 
$\tilde{T} \equiv T / m$, and $\tilde{R} \equiv R / I$

$$
\begin{aligned}
& \left\langle(\mathbf{v} \cdot \boldsymbol{\omega})^{2}\right\rangle^{(0)}=-4 \tilde{\nu} \tilde{T} \tilde{R} \\
& \left\langle(\mathbf{V} \cdot \boldsymbol{\omega})^{2}\right\rangle^{(0)}=-3 \tilde{\nu} \tilde{T} \tilde{R} \\
& \left\langle(\hat{\mathbf{n}} \cdot \mathbf{v})^{2}(\hat{\mathbf{n}} \cdot \boldsymbol{\omega})^{2}\right\rangle^{(0)}=-2 \tilde{\nu} \tilde{T} \tilde{R} \\
& \left\langle(\hat{\mathbf{n}} \cdot \mathbf{V})^{2}(\hat{\mathbf{n}} \cdot \boldsymbol{\omega})^{2}\right\rangle^{(0)}=-\tilde{\nu} \tilde{T} \tilde{R} \\
& \left\langle[(\hat{\mathbf{n}} \times \boldsymbol{\Omega}) \cdot \boldsymbol{\omega}]^{2}\right\rangle^{(0)}=-2 \tilde{\nu} \tilde{R}^{2} \\
& \left\langle[(\hat{\mathbf{n}} \times \mathbf{v}) \cdot \mathbf{V}]^{2}\right\rangle^{(0)}=-2 \tilde{\nu} \tilde{T}^{2} \\
& \langle(\hat{\mathbf{n}} \cdot \mathbf{v})(\hat{\mathbf{n}} \cdot \boldsymbol{\omega})(\mathbf{v} \cdot \boldsymbol{\omega})\rangle^{(0)}=-2 \tilde{\nu} \tilde{T} \tilde{R} \\
& \langle(\hat{\mathbf{n}} \cdot \mathbf{V})(\hat{\mathbf{n}} \cdot \boldsymbol{\omega})(\mathbf{V} \cdot \boldsymbol{\omega})\rangle^{(0)}=-\tilde{\nu} \tilde{T} \tilde{R} \\
& \left\langle\mathbf{v}^{2}(\hat{\mathbf{n}} \times \boldsymbol{\Omega})^{2}\right\rangle^{(0)}=-8 \tilde{\nu} \tilde{T} \tilde{R} \\
& \left\langle\mathbf{V}^{2}(\hat{\mathbf{n}} \times \boldsymbol{\Omega})^{2}\right\rangle^{(0)}=-6 \tilde{\nu} \tilde{T} \tilde{R} \\
& \left\langle\mathbf{v}^{2}(\hat{\mathbf{n}} \times \mathbf{v})^{2}\right\rangle^{(0)}=-12 \tilde{\nu} \tilde{T}^{2} \\
& \left\langle\mathbf{V}^{2}(\hat{\mathbf{n}} \times \mathbf{v})^{2}\right\rangle^{(0)}=-6 \tilde{\nu} \tilde{T}^{2} \\
& \left\langle\mathbf{v}^{2} \boldsymbol{\omega}^{2}\right\rangle^{(0)}=-12 \tilde{\nu} \tilde{T} \tilde{R} \\
& \left\langle(\hat{\mathbf{n}} \cdot \mathbf{v})^{2} \boldsymbol{\omega}^{2}\right\rangle^{(0)}=-6 \tilde{\nu} \tilde{T} \tilde{R} \\
& \left\langle(\hat{\mathbf{n}} \cdot \mathbf{v})^{2}(\hat{\mathbf{n}} \times \boldsymbol{\Omega})^{2}\right\rangle^{(0)}=-4 \tilde{\nu} \tilde{T} \tilde{R} \\
& \left\langle[(\hat{\mathbf{n}} \times \mathbf{v}) \cdot \boldsymbol{\Omega}]^{2}\right\rangle^{(0)}=-2 \tilde{\nu} \tilde{T} \tilde{R} \\
& \left\langle(\hat{\mathbf{n}} \cdot \mathbf{v})^{2}(\hat{\mathbf{n}} \times \mathbf{v})^{2}\right\rangle^{(0)}=-4 \tilde{\nu} \tilde{T}^{2} \\
& \left\langle(\hat{\mathbf{n}} \times \boldsymbol{\Omega})^{2} \boldsymbol{\omega}^{2}\right\rangle^{(0)}=-6 \tilde{\nu} \tilde{R}^{2} \\
& \left\langle(\hat{\mathbf{n}} \times \boldsymbol{\Omega})^{2} \boldsymbol{\Omega}^{2}\right\rangle^{(0)}=-10 \tilde{\nu} \tilde{R}^{2} \\
& \left\langle(\hat{\mathbf{n}} \times \boldsymbol{\Omega})^{4}\right\rangle^{(0)}=-8 \tilde{\nu} \tilde{R}^{2} \\
& \left\langle(\hat{\mathbf{n}} \times \boldsymbol{\Omega})^{2}(\hat{\mathbf{n}} \times \mathbf{v})^{2}\right\rangle^{(0)}=-4 \tilde{\nu} \tilde{T} \tilde{R} \\
& \left\langle(\mathbf{v} \cdot \boldsymbol{\omega})^{2}\right\rangle^{\text {even }}=-24 \tilde{\nu} \tilde{T}^{2} \tilde{R}^{2} \\
& \left\langle(\mathbf{V} \cdot \boldsymbol{\omega})^{2}\right\rangle^{\text {even }}=-15 \tilde{\nu} \tilde{T}^{2} \tilde{R}^{2} \\
& \left\langle(\hat{\mathbf{n}} \cdot \mathbf{v})^{2}(\hat{\mathbf{n}} \cdot \boldsymbol{\omega})^{2}\right\rangle^{\text {even }}=-6 \tilde{\nu} \tilde{T}^{2} \tilde{R}^{2} \\
& \left\langle(\hat{\mathbf{n}} \cdot \mathbf{V})^{2}(\hat{\mathbf{n}} \cdot \boldsymbol{\omega})^{2}\right\rangle^{\text {even }}=-3 \tilde{\nu} \tilde{T}^{2} \tilde{R}^{2} \\
& \left\langle[(\hat{\mathbf{n}} \times \boldsymbol{\Omega}) \cdot \boldsymbol{\omega}]^{2}\right\rangle^{\text {even }}=2 \tilde{\nu} \tilde{T} \tilde{R}^{3} \\
& \langle(\hat{\mathbf{n}} \cdot \mathbf{v})(\hat{\mathbf{n}} \cdot \boldsymbol{\omega})(\mathbf{v} \cdot \boldsymbol{\omega})\rangle^{\text {even }}=-12 \tilde{\nu} \tilde{T}^{2} \tilde{R}^{2} \\
& \langle(\hat{\mathbf{n}} \cdot \mathbf{V})(\hat{\mathbf{n}} \cdot \boldsymbol{\omega})(\mathbf{V} \cdot \boldsymbol{\omega})\rangle^{\text {even }}=-6 \tilde{\nu} \tilde{T}^{2} \tilde{R}^{2} \\
& \left\langle\mathbf{v}^{2}(\hat{\mathbf{n}} \times \boldsymbol{\Omega})^{2}\right\rangle^{\text {even }}=6 \tilde{\nu} \tilde{T}^{2} \tilde{R}^{2}
\end{aligned}
$$




$$
\begin{aligned}
\left\langle\mathbf{V}^{2}(\hat{\mathbf{n}} \times \boldsymbol{\Omega})^{2}\right\rangle^{\text {even }}= & 3 \tilde{\nu} \tilde{T}^{2} \tilde{R}^{2} \\
\left\langle(\hat{\mathbf{n}} \cdot \mathbf{v})^{2}(\hat{\mathbf{n}} \times \boldsymbol{\Omega})^{2}\right\rangle^{\text {even }}= & =6 \tilde{\nu} \tilde{T}^{2} \tilde{R}^{2} \\
\left\langle[(\hat{\mathbf{n}} \times \mathbf{v}) \cdot \boldsymbol{\Omega}]^{2}\right\rangle^{\text {even }}= & =6 \tilde{\nu} \tilde{T}^{2} \tilde{R}^{2} \\
\left\langle\boldsymbol{\omega}^{2}(\hat{\mathbf{n}} \times \boldsymbol{\Omega})^{2}\right\rangle^{\text {even }}= & =3 \tilde{\nu} \tilde{T} \tilde{R}^{3} \\
\left\langle\boldsymbol{\Omega}^{2}(\hat{\mathbf{n}} \times \boldsymbol{\Omega})^{2}\right\rangle^{\text {even }}= & 7 \tilde{\nu} \tilde{T} \tilde{R}^{3} \\
\left\langle(\hat{\mathbf{n}} \times \boldsymbol{\Omega})^{4}\right\rangle^{\text {even }}= & 8 \tilde{\nu} \tilde{T} \tilde{R}^{3} \\
\left\langle(\hat{\mathbf{n}} \times \mathbf{v})^{2}(\hat{\mathbf{n}} \times \boldsymbol{\Omega})^{2}\right\rangle^{\text {even }}= & 0 \\
\langle(\mathbf{v} \cdot \boldsymbol{\omega})(\mathbf{V} \cdot \boldsymbol{\Omega})\rangle^{\text {odd }} & =-20 \tilde{\nu} \tilde{T}^{2} \tilde{R}^{2} \\
\langle(\hat{\mathbf{n}} \cdot \mathbf{v})(\hat{\mathbf{n}} \cdot \boldsymbol{\omega})(\mathbf{V} \cdot \boldsymbol{\Omega})\rangle^{\text {odd }} & =-10 \tilde{\nu} \tilde{T}^{2} \tilde{R}^{2} \\
\langle(\hat{\mathbf{n}} \cdot \mathbf{V})(\hat{\mathbf{n}} \cdot \boldsymbol{\Omega})(\mathbf{v} \cdot \boldsymbol{\omega})\rangle^{\text {odd }} & =-7 \tilde{\nu} \tilde{T}^{2} \tilde{R}^{2} \\
\langle(\hat{\mathbf{n}} \cdot \mathbf{v})(\hat{\mathbf{n}} \cdot \mathbf{V})(\hat{\mathbf{n}} \cdot \boldsymbol{\omega})(\hat{\mathbf{n}} \cdot \boldsymbol{\Omega})\rangle^{\text {odd }} & =-4 \tilde{\nu} \tilde{T}^{2} \tilde{R}^{2} \\
\langle[(\hat{\mathbf{n}} \times \mathbf{v}) \cdot \mathbf{V}][(\hat{\mathbf{n}} \times \boldsymbol{\Omega}) \cdot \boldsymbol{\omega}]\rangle^{\text {odd }} & =0 \\
\langle(\hat{\mathbf{n}} \cdot \boldsymbol{\omega})(\hat{\mathbf{n}} \cdot \boldsymbol{\Omega})(\mathbf{v} \cdot \mathbf{V})\rangle^{\text {odd }} & =-2 \tilde{\nu} \tilde{T}^{2} \tilde{R}^{2} \\
\langle[(\hat{\mathbf{n}} \times \mathbf{v}) \cdot \boldsymbol{\omega}][(\hat{\mathbf{n}} \times \boldsymbol{\Omega}) \cdot \mathbf{V}]\rangle^{\text {odd }} & =-5 \tilde{\nu} \tilde{T}^{2} \tilde{R}^{2}
\end{aligned}
$$

\section{B Correction terms for the temperatures}

To calculate $\mathrm{d} T / \mathrm{d} t=\left\langle i \mathcal{L}_{+} T\right\rangle_{t}$ one essentially proceeds along the same lines of reasoning as detailed above. First of all, it is again advantageous to write the corrections to the Gaussian distribution function explicitly, that is,

$$
\left\langle i \mathcal{L}_{+} T\right\rangle_{t}=\left\langle\left(\hat{b}_{12}-1\right) T\right\rangle^{(0)}+b(t)\left\langle\left(\hat{b}_{12}-1\right) T\right\rangle^{(1)}
$$

where

$$
\begin{aligned}
\frac{3}{4 m}\left(\hat{b}_{12}-1\right) T= & \eta_{t}\left(\eta_{t}-1\right)(\hat{\mathbf{n}} \times \mathbf{v})^{2}+\eta_{n}\left(\eta_{n}-1\right)(\hat{\mathbf{n}} \cdot \mathbf{v})^{2}+\eta_{t}^{2} a^{2}(\hat{\mathbf{n}} \times \boldsymbol{\Omega})^{2} \\
& +\eta_{t}\left(2 \eta_{t}-1\right) a \mathbf{v} \cdot(\hat{\mathbf{n}} \times \boldsymbol{\Omega})
\end{aligned}
$$

and

$$
\frac{3}{4 m}\left(\hat{b}_{12}-1\right) R=\frac{\eta_{t}^{2}}{q}(\hat{\mathbf{n}} \times \mathbf{v})^{2}+\eta_{t}\left(\frac{\eta_{t}}{q}+1\right) a^{2}(\hat{\mathbf{n}} \times \boldsymbol{\Omega})^{2}+\eta_{t}\left(\frac{2 \eta_{t}}{q}+1\right) a \mathbf{v} \cdot(\hat{\mathbf{n}} \times \boldsymbol{\Omega}) .
$$

The term $\left\langle\left(\hat{b}_{12}-1\right) T\right\rangle^{(0)}$ is already known [25] and the only other contribution is

$$
\left\langle(\hat{\mathbf{n}} \times \boldsymbol{\Omega})^{2}\right\rangle^{\text {even }}=2 \tilde{\nu} \tilde{T} \tilde{R}^{2}
$$




\section{References}

1. I. Goldhirsch, Ann. Rev. Fluid Mech. 35, 267 (2003)

2. A. Levy, H. Kalman, Handbook of Conveying and Handling of Particulate Solids (Elsevier, Amsterdam, 2001)

3. R. Greenberg, A. Brahic, eds., Planetary Rings (Arizona Univ. Press., Tucson, 1984)

4. T. Pöschel, S. Luding, eds., Granular Gases, Lecture Notes in Physics, Vol. 564 (Springer, Berlin, 2001)

5. T. Pöschel, N.V. Brilliantov, eds., Granular Gas Dynamics, Lecture Notes in Physics, Vol. 624 (Springer, Berlin, 2003)

6. N.V. Brilliantov, T. Pöschel, Kinetic Theory of Granular Gases (Oxford University Press, Oxford, 2004)

7. S. McNamara, W.R. Young, Phys. Fluids A 4, 496 (1992)

8. I. Goldhirsch, G. Zanetti, Phys. Rev. Lett. 70, 1619 (1993)

9. R. Brito, M.H. Ernst, Europhys. Lett. 43, 497 (1998)

10. A. Goldshtein, M. Shapiro, J. Fluid Mech. 282, 75 (1995)

11. T.P.C. van Noije, M.H. Ernst, Granular Matter 1, 57 (1998)

12. S.E. Esipov, T. Pöschel, J. Stat. Phys. 86, 1385 (1997)

13. J.J. Brey, D. Cubero, M.J. Ruiz-Montero, Phys. Rev. E 59, 1256 (1999)

14. P. Deltour, J.L. Barrat, J. Phys. I (France) 7, 137 (1997)

15. M. Huthmann, J. Orza, R. Brito, Granular Matter 2, 189 (2000)

16. N.V. Brilliantov, T. Pöschel, Phys. Rev. E 61, 2809 (2000)

17. I. Goldhirsch, H.S. Noskowicz, O. Bar-Lev, The Homogeneous Cooling State Revisited, in [5], p. 37

18. T. Pöschel, N.V. Brilliantov, A. Formella, Phys. Rev. E 74, 041302 (2006)

19. J.J. Brey, M.J. Ruiz-Montero, R. Garcia-Rojo, Phys. Rev. E 60, 7174 (1999)

20. J.J. Brey, M.J. Ruiz-Montero, D. Cubero, R. Garcia-Rojo, Phys. Fluids 12, 876 (2000)

21. N.V. Brilliantov, T. Pöschel, Phys. Rev. E 61, 1716 (2000)

22. A. Santos, J.W. Dufty, Phys. Rev. Lett. 86, 4823 (2001)

23. V. Garzo, J.M. Montanero, Phys. Rev. E 69, 021301 (2004)

24. M. Huthmann, A. Zippelius, Phys. Rev. E 56, R6275 (1997)

25. T. Aspelmeier, M. Huthmann, A. Zippelius, Free Cooling of Particles with Rotational Degrees of Freedom, in [4], p. 31

26. I. Goldhirsch, S.H. Noskowicz, O. Bar-Lev, J. Phys. Chem. 109, 21449 (2005)

27. T. Elperin, E. Golshtein, Physica A 247, 67 (1997)

28. J.T. Jenkins, M.W. Richman, Phys. Fluids 28, 3485 (1985)

29. C.K.K. Lun, S.B. Savage, J. Appl. Mech. Trans. ASME 54, 47 (1987)

30. H.M. Jaeger, C. Liu, S.R. Nagel, T.A. Witten, Europhys. Lett. 11, 619 (1990)

31. S. Luding, Phys. Rev. E 52, 3416 (1995)

32. J.T. Jenkins, M. Louge, Phys. Fluids 9, 2835 (1997)

33. S.G. Bardenhagen, J.U. Brackbill, D. Sulsky, Phys. Rev. E 62, 3882 (2000)

34. R. Cafiero, S. Luding, H.J. Herrmann, Europhys. Lett. 60, 854 (2002)

35. N. Mitarai, H. Hayakawa, H. Nakanishi, Phys. Rev. Lett. 88, 174301 (2002)

36. I. Goldhirsch, S.H. Noskowicz, O. Bar-Lev, Phys. Rev. Lett. 95, 068002 (2005)

37. N.V. Brilliantov, T. Pöschel, W.T. Kranz, A. Zippelius, Phys. Rev. Lett. 98, 128001 (2007)

38. N.V. Brilliantov, F. Spahn, J.M. Hertzsch, T. Pöschel, Phys. Rev. E 53, 5382 (1996)

39. T. Schwager, T. Pöschel, Phys. Rev. E 57, 650 (1998)

40. R. Ramírez, N.V. Brilliantov, T. Schwager, T. Pöschel, Phys. Rev. E 60, 4465 (1999)

41. V. Becker, T. Schwager, T. Pöschel, Phys. Rev. E 77, 011304 (2008)

42. S. Luding, M. Huthmann, S. McNamara, A. Zippelius, Phys. Rev. E 58, 3416 (1998)

43. T. Pöschel, N.V. Brilliantov, T. Schwager, Int. J. Mod. Phys. C 13, 1263 (2003)

44. S.H. Noskowicz, O. Bar-Lev, D. Serero, I. Goldhirsch, Europhys. Lett. 79, 60001 (2007)

45. G.A. Bird, Molecular Gas Dynamics and the Direct Simulation of Gas Flows (Oxford University Press, 1994)

46. T. Pöschel, T. Schwager, Computational Granular Dynamics (Springer, New York, 2005)

47. J.J. Brey, D. Cubero, in Granular Gases, edited by T. Pöschel, S. Luding, Lecture Notes in Physics, Vol. 564 (Springer, Berlin, 2001), p. 59

48. B. Gayen, M. Alam, Phys. Rev. Lett. 100, 068002 (2008) 